\title{
Modelling the Galactic interstellar extinction distribution in three dimensions ${ }^{\star}$
}

\author{
D. J. Marshall ${ }^{1}$, A. C. Robin ${ }^{1}$, C. Reylé ${ }^{1}$, M. Schultheis ${ }^{1}$, and S. Picaud ${ }^{1,2}$ \\ 1 CNRS UMR 6091, Observatoire de Besançon, BP 1615, 25010 Besançon Cedex, France \\ e-mail: marshall@obs-besancon.fr \\ 2 Instituto de Astronomia e Geofísica (IAG/USP), São Paulo, Brazil
}

Received 18 July 2005 / Accepted 25 March 2006

\section{ABSTRACT}

\begin{abstract}
Aims. The Two Micron All Sky Survey, along with the Stellar Population Synthesis Model of the Galaxy, developed in Besançon, is used to calculate the extinction distribution along different lines of sight. By combining many lines of sight, the large scale distribution of interstellar material can be deduced.

Methods. The Galaxy model is used to provide the intrinsic colour of stars and their probable distances, so that the near infrared colour excess, and hence the extinction, may be calculated and its distance evaluated. Such a technique is dependent on the model used, however we are able to show that moderate changes in the model parameters result in insignificant changes in the predicted extinction.

Results. This technique has now been applied to over 64000 lines of sight, each separated by $15^{\prime}$, in the inner Galaxy $\left(|l| \leq 100^{\circ}\right.$, $|b| \leq 10^{\circ}$ ). We have projected our three dimensional results onto a two dimensional plane in order to compare them with existing two dimensional extinction maps and CO surveys. We find that although differences exist due to the different methods used or the medium traced, the same large scale structures are visible in each of the different maps. Using our extinction map, we have derived the main characteristics of the large scale structure of the dust distribution. The scale height of the interstellar matter is found to be $125_{-7}^{+17} \mathrm{pc}$. The dust distribution is found to be asymmetrically warped, in agreement with CO and HI observations of the ISM. However, the slope of the dust warp and the galactocentric distance where it starts are found to be smaller than the values measured for the external $\mathrm{HI}$ disc: for positive longitudes the angle is $\theta=89^{\circ}$, it starts at $8.7 \mathrm{kpc}$ from the Galactic center and grows with a slope of 0.14 , while at negative longitudes, the angle of the maximum is at $\theta=272^{\circ}$, the starting radius $7.8 \mathrm{kpc}$ and the slope 0.11 . Finally, the presence of dust is detected in the Galactic bulge. It forms an elongated structure approximately $5.2 \mathrm{kpc}$ long and lies at an angle of $30 \pm 5^{\circ}$ with respect to the Sun-Galactic centre direction. This may be interpreted as a dust lane along the Galactic bar. This resulting extinction map will be useful for studies of the inner Galaxy and its stellar populations.
\end{abstract}

Key words. ISM: dust, extinction - ISM: structure - Galaxy: structure

\section{Introduction}

Interstellar extinction remains a serious obstacle for the observation of stars in the Milky Way, and for interpreting these observations in terms of Galactic structure. It has two main effects, that of dimming sources and reddening them. Any stellar study near the Galactic plane would need to correct for this effect to enable a proper interpretation of the observations. Recent infrared surveys provide a superb tool to probe further into the densest parts of the Galaxy as the extinction suffered in the $K_{\mathrm{s}}$ band is approximately one tenth of that suffered in the visible.

Furthermore, extragalactic studies often shun the so called "Zone of Avoidance" at around $|b| \lesssim 10^{\circ}$ and towards the inner Galaxy due to the amount of extinction and the high source confusion. The determination of interstellar extinction in this zone, which covers about $10 \%$ of the infrared sky, is thus an

* The three dimensional results (Table 1) are available in electronic form at the CDS via anonymous ftp to cdsarc.u-strasbg.fr (130.79.128.5) or via http://cdsweb.u-strasbg.fr/cgi-bin/ qcat?J/A+A/453/635 invaluable step to identifying low extinction windows at low Galactic latitude.

Several studies have been undertaken to further our knowledge of the distribution of the extinction in our Galaxy. Most of them provide a two dimensional map of the extinction in different regions of the Galaxy in which they calculate either the mean extinction or the total extinction for various lines of sight: Froebrich et al. (2005) use accumulative star counts from the 2MASS survey to construct a relative extinction map of the Galactic plane; Sumi (2004) has created a two dimensional map of the mean extinction towards the Galactic bulge using red clump giants from the Optical Gravitational Lensing Experiment II; Dutra et al. (2003) and Schultheis et al. (1999) determined the mean extinction towards the same region using 2MASS data and DENIS data, respectively; Schlegel et al. (1998) used the dust IR emission as observed by DIRBE to create an all sky two dimensional map of the total extinction integrated along each line of sight. However, according to Arce et al. (1999), the Schlegel et al. (1998) map overestimates the reddening by a factor of $1.3-1.5$ in regions of smooth extinction with $A_{\mathrm{V}}>0.5$, and underestimates it in regions of steep 
extinction gradients. Cambrésy et al. (2005) also noticed that the correlation of the near-infrared extinction to the far-infrared optical depth shows a discrepancy of about $30 \%$ for $A_{V}>1$ mag, probably due to the ubiquitous presence of fluffy grains.

Other authors have determined the distribution of extinction along different lines of sight using various methods: Fitzgerald (1968) provided the local distribution of extinction up to a few kiloparsecs using photoelectric photometry. Later on, Neckel et al. (1980) measured extinction and distances towards individual $\mathrm{O}, \mathrm{B}$ and $\mathrm{A}$ stars. These studies provide limited resolution for the extinction distribution due to the scarceness of the distribution of hot stars, often related to clouds. They do not provide the distribution of the diffuse extinction. The study of Berdnikov et al. (1990) is limited to a sample of 615 stars in 9 Kapteyn Selected Areas and shows considerable dispersion in extinction among the stars in a given direction. Arenou et al. (1992) mapped the local extinction from a collection of spectral and photometric data. The validity of this map is limited to a distance of about $1 \mathrm{kpc}$, hence it cannot be used at $|b|<10^{\circ}$, where a significant part of the extinction is situated at distances larger than $1 \mathrm{kpc}$. In addition, the resolution of the map is of the order of several degrees. Cambrésy (1999a) determined detailed maps of extinction in nearby Giant Molecular Clouds from USNO star counts, which are very useful for studies of these clouds, but not outside. He also considered the whole sky extinction from USNO star counts, but still with a two dimensional approach, and with a limited spatial resolution of $6^{\circ}$ (Cambrésy 1999b). A great deal of other determinations can also be found in the literature which concern one or a few lines of sight. Putting all these maps together leads to inhomogeneous maps with poorly defined accuracy. They also have the problem of matching different results at the boundaries between authors (Hakkila et al. 1997).

The relationship between infrared colour excess and extinction is well documented, and has been in use for some time (Jones et al. 1984; Lada et al. 1994). In this type of study, stars that are reddened due to their location behind a cloud are compared to nearby, supposedly un-reddened, stars. Our approach is to compare observed infrared colours with those from the Besançon model of the Galaxy. Using this technique, with which we are able to calculate the extinction along the line of sight, we have created a map of $K_{\mathrm{s}}$ band extinction $\left(A_{K \mathrm{~s}}\right)$ in the plane of the inner Galaxy $\left(|l|<100^{\circ},|b|<10^{\circ}\right)$ and in three dimensions. Our method for determining extinction does not assume any model for the dust distribution. Instead, we compare the difference in the $J-K_{\mathrm{s}}$ colour distribution of stars from the Besançon model of the Galaxy (Robin et al. 2003) to those from 2MASS observations (Cutri et al. 2003); the difference between the two is then attributed to interstellar extinction. The $J-K_{\mathrm{s}}$ colour index provides an excellent probe of interstellar extinction, in the Galactic plane where it is high, as radiation at two microns is less absorbed than visible light by around an order of magnitude. This technique can therefore provide a global picture of the three dimensional distribution of interstellar dust.

Our use of the 2MASS data is discussed in Sect. 2, the generation of catalogues of stars using the Besançon model of the Galaxy in Sect. 3. In Sect. 4 we describe how we transform a difference in colour between observed and modelled stars into a prediction of the three dimensional extinction along a line of sight. The three dimensional results are presented in Sect. 5; these are compared to observations of $\mathrm{CO}$ and to other extinction data, and the large scale characteristics of its distribution are discussed. The sensitivity of the results to changes in model parameters is estimated and limits and possible bias are discussed in Sect. 6. Our results are summarised in Sect. 7.

\section{2MASS point source catalogue}

\subsection{Description of the survey}

The Two Micron All Sky Survey (2MASS) is a ground based survey which uniformly scanned the entire sky in three nearinfrared bands $\left(J, H\right.$ and $\left.K_{\mathrm{s}}\right)$. Amongst its final products is the point source catalogue (PSC) which includes point sources brighter than about $1 \mathrm{mJy}$ in each band, with signal-to-noise ratio (SNR) greater than 10, using a pixel size of $2.0^{\prime \prime}$. Each star has accurate photometry and astrometry as defined in the level 1 requirements (Cutri et al. 2003).

The quality of the data was destined to meet or surpass all of the level 1 requirements. We list three of them below:

- photometric sensitivity of $10-\sigma$ at $15.8,15.1,14.3$ mag at $J$, $H, K_{\mathrm{s}}$ respectively for $|b|>10^{\circ}$;

- completeness $>0.99$ at $10-\sigma$ sensitivity limits;

- reliability $>0.9995$.

These requirements were met for the entire unconfused sky. However, the 2MASS survey has no level 1 requirements for the Galactic Plane - these specifications apply only to high Galactic latitudes. Within approximately a $6^{\circ}$ radius of the Galactic centre, the average magnitude for a $S N R=10$ source is up to $\sim 2 \mathrm{mag}$ brighter than the typical $S N R=10$ source in unconfused regions. This is also seen for various regions in the Galactic plane at $|b| \lesssim$ $3^{\circ}$ and $\pm 60^{\circ}$ from the Galactic centre.

As well as containing default magnitudes and associated uncertainty for each star, the 2MASS PSC also contains a number of flags to aid the user in selecting stars appropriate for the study. We make use of the Read Flag (rd_flg), which indicates the method used to determine the default magnitude of the source, and the Photometry Quality Flag (ph_flg) which gives an indication of the quality of the photometric determination (signal to noise ratio, measurement error). More information on the different flags and their meanings can be found in Cutri et al. (2003).

The final 2MASS project also includes an extended source catalogue (XSC). By using a time varying PSF as well as the $J-K_{\mathrm{s}}$ and $H-K_{\mathrm{s}}$ colour of the sources, Cutri et al. (2003) were able to separate the extended sources from the point sources. After this classification, the final 2MASS PSC contains only point sources with a reliability of over $98 \%$.

\subsection{Completeness of the 2MASS PSC}

We are interested in regions in the Galactic Plane, where the completeness varies as a function of stellar density and can be as much as two magnitudes brighter than high latitude fields. The PSC can be compared quantitatively with model simulations only where the former is shown to be complete. As such, we must compute the completeness limit field by field.

The 2MASS definition of completeness is "the faintest magnitude bin which recovers $99 \%$ of the expected source counts". However, for their atlas images, Cutri et al. (2003) suggest another method, described below, which we have adopted. Star count histograms are constructed for each field at 0.2 mag intervals for point sources with magnitudes between 9 and 18 mag and where a reliable estimate of the photometric error could be determined in the appropriate band. This latter condition excludes only a few percent of the total number of stars in a field except in high confusion fields, for example near the bulge or 
in the Galactic plane, where as many as $20 \%$ of the stars in the 2MASS PSC may not have an associated photometric error. The completeness in a particular field is defined to be the bin that contains the highest star count. Stars detected by 2MASS but with photometry so poor that the error could not be determined are not used for the calculation of the limiting magnitude. This ensures that we do not overestimate the completeness of the observed stars which would introduce a large number of modelled stars with no observational counterpart.

\subsection{MASS selection criteria}

As stated, there are a number of flags for each star in the PSC in order to select them based on the quality of their photometry, or to reject them due to the risk that they are contaminated by nearby bright objects. However, if we are too severe with our selection we will reject valid stellar detections. We will be comparing the selected stars with the Besançon model; it thus follows that we must select all point sources from the PSC that correspond to actual stellar detections.

As we are interested in using the difference in observed and modelled $J-K_{\mathrm{s}}$ colour, we require detections in these two bands in order to admit a star into our calculation. However, a point source that has been detected in the $J$ and $K_{\mathrm{s}}$ bands without a corresponding detection in the $H$ band is not likely a valid stellar detection. Therefore, we restrict our selection to stars that have been detected in all three bands (2MASS rd_flag $\neq 0$ ). Stars above the completeness limit in either the $J, H$ or $K_{\mathrm{s}}$ bands are also rejected. We use the $J-K_{\mathrm{s}}$ colour index as it is less sensitive to metallicity and gravity than the $H-K_{\mathrm{s}}$ or $J-H$ colour indices.

This "full" catalogue includes all detected stars in the particular 2MASS field, even those with less accurate photometry. However, as they are real detections they must be included in our comparison with the Besançon model.

\subsection{Missing fields in the PSC}

The 2MASS catalogue contains a small number of "physical" or "effective" gaps, due either to unscanned areas of the sky or the removal of valid sources near tile boundaries, respectively. The total area of these gaps in our results amounts to less than 0.04 square degrees.

Other gaps in the 2MASS catalogue, which add up to a larger area in our results, include regions where bright stars have been removed, effectively creating a hole in the stellar density. All stars within the radius of the removed star are then excluded from the field of view; as these "missing" stars sample all stellar populations and distances, they do not introduce any bias or error into our extinction determination.

\section{The Galactic model}

\subsection{Description of the model}

The stellar population synthesis model of the Galaxy constructed in Besançon (Robin et al. 2003), hereafter called the Galactic model, is able to simulate the stellar content of the Galaxy by modelling four distinct stellar populations: the thin disc, the thick disc, the outer bulge and the spheroid. It also takes into account the dark halo and a diffuse component of the interstellar medium. It can be used to generate stellar catalogues for any given direction, and returns information on each star such as magnitude, colour, and distance as well as kinematics and other stellar parameters.
The approach of the Galactic model is semi-empirical as it is based on theoretical grounds (for example stellar evolution, galactic evolution and galactic dynamics) and is constrained by empirical observations (the local luminosity function for example). The Galactic potential is calculated in order to selfconsistently constrain the disc scale height. In addition, the model includes observational errors and Poisson noise to make it ideal for direct comparison with observations.

The Galactic model has been developed to return results in the near-infrared and visible filters. It has been shown to reproduce the stellar content in the infrared to a high degree of accuracy (see Sect. 6.2), and is a powerful tool to extract the extinction information embedded in the 2MASS observations.

\subsection{Model parameters}

In this study, limited to the inner Galaxy, the bulge and thin disc are the dominant populations. Hence, the thick disc and spheroid model parameters will not be described here, but can be found in Robin et al. (2003).

\subsubsection{Thin disc}

The thin disc is described by an evolutionary scheme with a two-slopes initial mass function and a constant star formation rate over the past 10 Gyr. The thin disc is divided into 7 age components, the first of which is called the young disc (age $<0.15$ Gyr). The other six components define the old disc.

The old thin disc density distribution is modelled using the Einasto (1979) law. The distribution of each old disc component is described by an axisymmetric ellipsoid with an axis ratio depending on the age; the density law is described by the subtraction of two ellipsoids:

$\rho_{\mathrm{d}}=\rho_{\mathrm{d} 0} \times[\operatorname{disc}-$ hole $]$

where

$$
\begin{aligned}
& \text { disc }=\exp \left(-\sqrt{0.25+\left(\frac{R}{R_{\mathrm{d}}}\right)^{2}+\left(\frac{Z}{\epsilon \times R_{\mathrm{d}}}\right)^{2}}\right) \\
& \text { hole }=\exp \left(-\sqrt{0.25+\left(\frac{R}{R_{\mathrm{h}}}\right)^{2}+\left(\frac{Z}{\epsilon \times R_{\mathrm{d}}}\right)^{2}}\right)
\end{aligned}
$$

and:

- $R$ and $Z$ are the cylindrical galactocentric coordinates;

$-\epsilon$ is the axis ratio of the ellipsoid. Recently revised axis ratios of the 6 age components of the old thin disc can be found in Robin et al. (2003);

- $R_{\mathrm{d}}$ is the scale length of the disc and is $2.510 \mathrm{kpc}$;

- $R_{\mathrm{h}}$ is the scale length of the hole and is $680 \mathrm{pc}$;

- the normalization $\rho_{\mathrm{d} 0}$ is deduced from the local luminosity function (Jahreiß \& Wielen 1997), assuming that the Sun is located at $R=8.5 \mathrm{kpc}$ and $Z=15 \mathrm{pc}$.

The two values for $R_{\mathrm{d}}$ and $R_{\mathrm{h}}$ are recent results from the method described in Picaud \& Robin (2004). The equation of the old disc differs from that found in Robin et al. (2003); the version presented here improves the modelling of the disc hole.

The young thin disc density distribution is:

$\rho_{\mathrm{d}}=\rho_{\mathrm{d} 0} \times\left[\exp \left(-\left(\frac{a}{R_{\mathrm{d}}}\right)^{2}\right)-\exp \left(-\left(\frac{a}{R_{\mathrm{h}}}\right)^{2}\right)\right]$ 
where $a^{2}=R^{2}+\left(\frac{Z}{\epsilon}\right)^{2}, R, Z$ and $\epsilon$ have the same significance as above and the scale lengths for the young disc are $R_{\mathrm{d}}=5 \mathrm{kpc}$ and $R_{\mathrm{h}}=3 \mathrm{kpc}$.

The stars from the young disc represent approximately $0.5 \%$ of the stars in this study, thus the impact of this population in our method is negligible.

\subsubsection{Outer Galactic bulge}

An analysis of DENIS data in the region with Galactic coordinates $-8^{\circ}<l<12^{\circ}$ and $|b|<4^{\circ}$ allowed Picaud \& Robin (2004) to constrain the shape of the triaxial bulge, as well as its age. The bulge is found to be boxy, prolate, with axis ratios 1:0.30:0.25, and oriented $10.6 \pm 3^{\circ}$ with respect to the Sun-centre direction. A full description of the parameter values of the bulge density law can be found in Picaud \& Robin (2004).

\subsubsection{Spiral structure and Galactic warp}

The version of the Galactic model we use here does not include any spiral structure. The stars that we will be using for our method, mostly old K\&M giants, are, according to the Galactic model, well over 1 Gyr old which corresponds to about 4 Galactic rotations. The effect of the spiral structure is thus assumed to be negligible for stars older than $1 \mathrm{Gyr}$.

The stellar warp is incorporated into the Galactic model as described in Robin et al. (2003). Here we give just the relevant values. The Galactic warp is modelled as a shift of the galactocentric coordinates perpendicular to the plane by a linear factor equal to 0.18 beyond a galactocentric distance of $8.4 \mathrm{kpc}$ (Derrière \& Robin 2001; Derrière 2001). This effect is maximum for an angle of $90^{\circ}$ from the Sun Galactic centre direction.

\subsection{Diffuse extinction}

At the time of writing, the Galactic model available online (http://www.obs-besancon.fr/modele/modele.html) is able to simulate interstellar extinction as diffuse extinction and as individual clouds. For the former, it is approximated using a double exponential disc with an ad hoc local normalisation (usually $0.7 \mathrm{mag} \mathrm{kpc}^{-1}$ for high latitude lines of sight). For the latter, any number of clouds can be added along the line of sight, defined by their distance and extinction $\left(A_{V}\right)$. A cloud covers the entire field of view and therefore affects all the stars at distances greater than it.

\subsection{Photometric errors in the model}

The 2MASS PSC supplies photometric errors for each band and for each star, where the conditions (atmospheric, crowding) permitted their determination. The Galactic model is able to simulate different photometric error laws to emulate the response of different detection methods (CCD, photographic plates, etc.). Photometric errors are included on each photometric band independently, the error being a function of the magnitude (as usually observed). An exponential function is able to reproduce typical CCD photometric errors very well.

The error law, as implemented in the model, is:

$\sigma(m)=A+\mathrm{e}^{(C \times m-B)}$

where $m$ is the magnitude in the band considered, and $A, B$ and $C$ are fitted to the set of data with which the model predictions are going to be compared. Figure 1 shows the 2MASS photometric

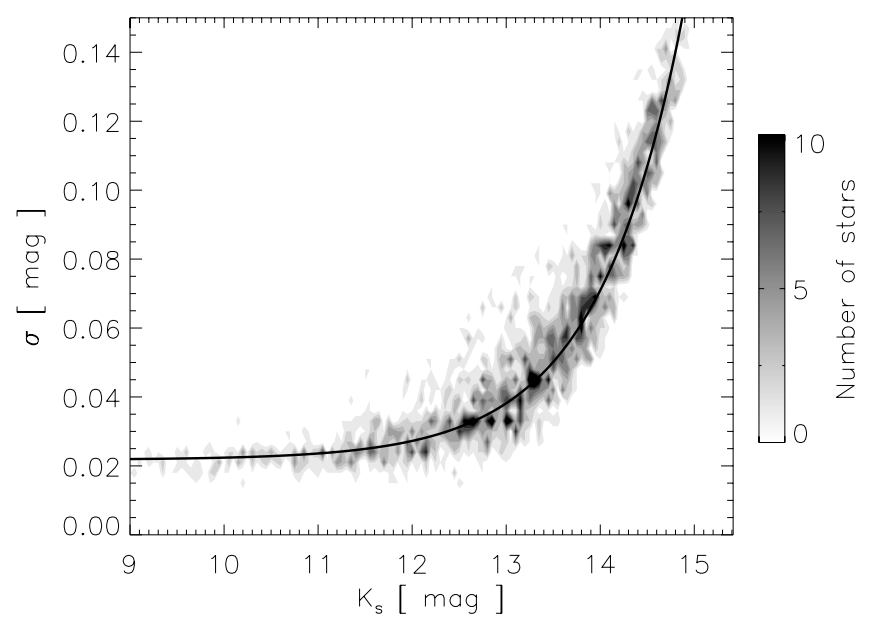

Fig. 1. Grey-scale representation of the photometric error as a function of the $K_{\mathrm{s}}$ magnitude for the 2MASS observations in the field $(l, b)=$ $\left(50^{\circ}, 0^{\circ}\right)$. The solid line shows our best fit using Eq. (3).

errors for the $K_{\mathrm{s}}$ band in the field $(l, b)=\left(50^{\circ}, 0^{\circ}\right)$. The solid line is the best fit using Eq. (3).

The photometric errors in the 2MASS PSC are not constant across the entire sky, as they depend on local atmospheric conditions and stellar density. To perform the fit for a given field, we select the 2MASS stars in that field where a reliable estimate of the photometric error could be determined in the appropriate band. The parameters of Eq. (3) are then determined by fitting the error law to the selected 2MASS stars. This enables us to define an average error with respect to apparent magnitude, field by field, for the stars in the 2MASS PSC.

\subsection{Limiting magnitudes in the model}

In order to closely model the 2MASS stars, we cut the modelled stars at the 2MASS completeness and at a $J, H$ and $K_{\mathrm{s}}$ magnitude of 9 as a lower limit. The faint limit ranges from $m_{K \mathrm{~s}} \sim$ 10.5 to $\sim 14.4$, and $m_{J} \sim 12.0$ to $\sim 15.8$, depending on the crowding of the field. As the magnitude increases, the reliability of the 2MASS catalogue in the Galactic plane decreases. Therefore, to ensure the we have the highest reliability we fix the faint magnitude limit of the $K_{\mathrm{s}}$ band to a maximum of 12 . The maximum magnitude used for the $J$ band, however, is chosen to be the completeness limit of the 2MASS observations in order to detect sources with high extinction.

\section{Extinction from 2 MASS and the Galaxy model}

Interstellar dust absorbs and scatters electromagnetic radiation, the effect being stronger for radiation at shorter wavelengths. This interstellar extinction results in observed stars appearing redder than they would in the absence of dust.

The Galactic model provides us with simulated, initially unreddened stars and the 2MASS PSC contains observed, reddened stars. If we assume that the Galactic model successfully predicts the average intrinsic colour and distance of stars in the Milky Way, then we may suppose that the difference between the modelled and observed stellar colour distributions is due solely to the effects of extinction.

The method that we detail in this section enables us to determine the extinction, as a function of distance and along any particular line of sight, which minimises the difference between the modelled and observed stellar colour distributions. 


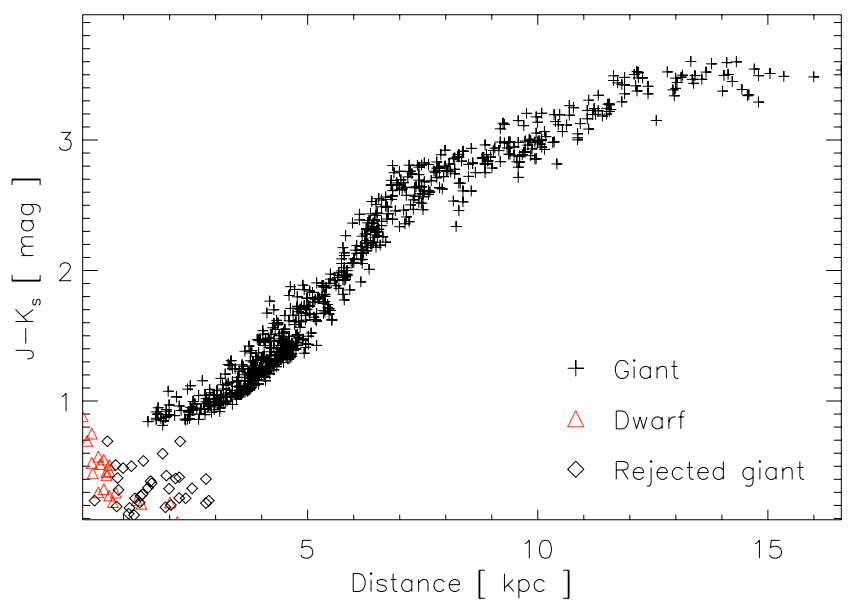

Fig. 2. Simulated colour-distance distribution towards $l=330^{\circ} \mathrm{b}=$ $1.5^{\circ}$. Crosses are giants, triangles are dwarf stars and the diamonds denote rejected giants (Sect. 4.3). The group of stars in the lower left corner is dominated by dwarfs and sub-giants. There is a relation between colour and distance for the giants, first dominated by K\&M stars, then, at larger distances by RGB/AGB stars.

\subsection{Colour distance relation}

In Fig. 2 we present an example field from the Galactic model towards $l=330^{\circ}, b=1.5^{\circ}$; the $J-K_{\mathrm{s}}$ distribution is clearly a function of distance. The dwarf and sub-giant populations are conspicuous on the bottom left of Fig. 2 as they do not follow the same colour distance relation as the regular giant population. Dwarf stars are observed locally; they are too faint to be seen at large distances. As such they do not suffer much interstellar reddening, so the reddest (faintest) dwarfs detected will, contrary to the giants, be the closest. These dwarf stars are removed from our stellar selection as described in Sect. 4.3. Most of the subgiants which are not on the same sequence as the giants are also removed in this process.

The colour distance relation for the giants holds due to the fact that in the Galactic Plane the stars that dominate the counts at $9<K_{\mathrm{s}}<14$ are mostly $\mathrm{K} 0$ to $\mathrm{K} 2$ giants and RGB/AGB giants; the latter dominate at larger distances due to their high luminosities. Stars which are homogeneous in intrinsic $J-K_{\mathrm{s}}$ colour and in absolute magnitude $M_{K}$ would have the same colour in a region with no extinction, showing a narrow histogram of colour. As extinction is an increasing function of distance, the $J-K_{\mathrm{s}}$ colour of these stars will naturally increase with distance.

In the following we show how we compute the extinction, and its distribution in distance along the line of sight, for a particular field. We use the Galactic model to simulate a variable proportion of $K$ and $M$ giants, as well as RGB/AGB stars, as a function of $K$ magnitude along each line of sight, resulting in a realistic $J-K_{\mathrm{s}}$ distribution. In this way, the colour distance property of giants can be used to translate the shape of a $J-K_{\mathrm{s}}$ histogram of 2 MASS stars in a given direction into a distribution of the extinction along the line of sight.

\subsection{NIR colour excess}

As mentioned above, interstellar dust has the effect of reddening starlight. Thus a difference in stellar colour (observed - intrinsic) can be used to deduce the amount of extinction the starlight has suffered. For a group of stars assumed to be at a similar distance, the difference between their mean intrinsic colour and their mean observed colour (the mean colour excess) yields the total mean extinction at that distance. Using a reddening law (for example Mathis 1990), we may write:

$\overline{A_{K \mathrm{~s}}}=0.67 \times \overline{E\left(J-K_{\mathrm{s}}\right)}$

where $A_{K \mathrm{~s}}$ is the extinction in the band $K_{\mathrm{s}}, E\left(J-K_{\mathrm{s}}\right)$ is the $J-K_{\mathrm{s}}$ colour excess and the bar denotes that we have taken the mean for the group of stars.

Instead of using the intrinsic colour of the stars as reference, we use simulated stellar colours from the Galactic model and we compute the difference in extinction between observed and modelled stars:

$\delta A_{K \mathrm{~s}}=0.67 \times\left[\left(\overline{J-K_{\mathrm{s}}}\right)_{\mathrm{obs}}-\left(\overline{J-K_{\mathrm{s}}}\right)_{\mathrm{sim}}\right]$

where the obs and sim subscripts denote observed and simulated colours, respectively. In other words, with Eq. (5) we are calculating the extinction necessary to reduce, as much as possible, the difference in colour between simulated and observed colour histograms. This equation may be used if the simulated stars have been reddened or not, as it simply translates the difference in colour between simulated and observed stars into a difference in extinction.

In order to calculate the extinction distribution as a function of distance for a particular line of sight and field size, we proceed as follows. We start by applying a simple extinction distribution, as explained in Sect. 3.3, to the simulated stars. The local normalisation is chosen in order to minimise the difference in the $J-K_{\mathrm{s}}$ colour distributions of the observed and simulated stars. The simulated, reddened stars are then cut at the faint magnitude limits for the field of the 2MASS observations. The dwarf stars, identified as explained below in Sect. 4.3, are removed from both the observations and the model.

Next, both the simulated and observed stars are sorted by increasing $J-K_{\mathrm{s}}$ colour; due to the colour distance relation mentioned above, this means that we are effectively sorting the stars by increasing distance. We then bin the simulated stars by colour; the number of stars in a bin is chosen such that the median distance of the simulated stars in each successive bin increases.

The observed stars are put into the same number of bins as the simulated stars, each bin containing the same relative number of stars:

$n_{\mathrm{obs}_{i}}=\frac{N_{\mathrm{obs}}}{N_{\mathrm{mod}}} n_{\bmod _{i}}$

where $N_{\text {obs }}$ and $N_{\text {mod }}$ are the total number of stars in the observations and model, respectively, and $n_{\mathrm{obs}_{i}}$ and $n_{\bmod _{i}}$ denote the number of stars in a particular bin for the observations and the Galactic model, respectively. We may then obtain the magnitude of the extinction by applying Eq. (5), bin by bin, and assuming that the extinction calculated is at the median distance of the simulated stars in that bin. Using the relative number of stars makes the method less sensitive to a difference in the number of stars between the Galactic model and the observations, hence to the assumed large scale structure of the stellar populations (Sect. 6.2).

\subsection{Removing the dwarf stars}

In Fig. 3 the colour magnitude diagram of the Galactic model for the line of sight $(l, b)=(330,1.5)$ is shown. The giant and dwarf populations occupy distinct regions in the diagram: the dwarf population is on the left hand side, the giants are on the right. The line running down the middle indicates the upper limit of dwarf 


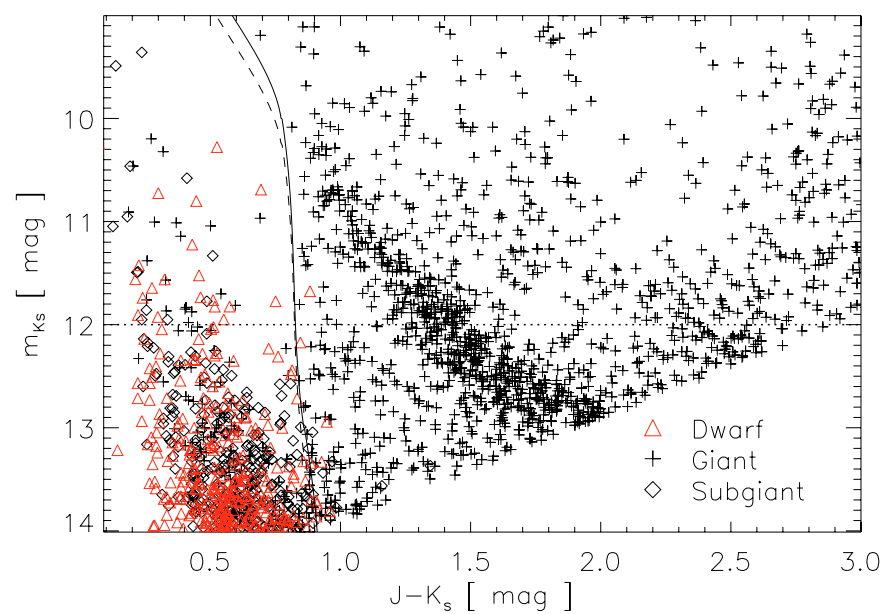

Fig. 3. Dwarf star removal for the field $(l, b)=(330,1.5)$. The triangles are dwarf stars, the diamonds are sub-giants and the crosses are regular giants. The lines running from top to bottom near $J-K_{\mathrm{s}}=0.9$ are the cut-off for dwarf stars before (dashed) and after (solid) the first determination of the extinction. The dotted line at $m_{K \mathrm{~s}}=12$ shows our faint magnitude cutoff.

$J-K_{\mathrm{s}}$ colour as a function of magnitude (described below). We select all stars redder than this upper limit; what will be left will be almost exclusively $\mathrm{K} \& \mathrm{M}$ giants and RGB/AGB giants.

For a given apparent magnitude, we are able to define a maximum $J-K_{\mathrm{s}}$ colour for the dwarf stars as follows. If we suppose that, on average, the closest stars are at $100 \mathrm{pc}$, then we can assign this distance to the reddest dwarf stars as these will be the closest. The absolute magnitude of these dwarf stars at a given apparent magnitude can then be calculated, initially assuming that they do not suffer any extinction. Using the main sequence relation, we are able to convert this absolute magnitude to a $J-K_{\mathrm{s}}$ colour index. This colour index thus defines the upper limit for the dwarf stars. However the dwarf stars do suffer some extinction; after an initial estimate of the extinction has been obtained using the giants (Sect. 4.2), we redden the upper limit used according to the extinction found at the distance of the dwarf stars. This gives us a new selection of giants with which we may recalculate the extinction. Figure 3 shows the $J-K_{\mathrm{s}}$ cut-off before (dashed line) and after (solid line) the first extinction determination. As can be seen, this is not a large shift as the dwarf stars are indeed local.

\subsection{Iterative method}

After determining the extinction distribution as described above we apply this extinction to the stars from the Galactic model for the particular line of sight. To test the resulting distribution we construct a $J-K_{\mathrm{s}}$ histogram of the adjusted model and the 2MASS observations (including the dwarf stars) - these can be compared by means of a $\chi^{2}$ test.

We compute the $\chi^{2}$ statistic for the two binned sets of data as detailed in Press et al. (1992):

$\chi^{2}=\sum_{i} \frac{\left(\sqrt{N_{\mathrm{obs}} / N_{\mathrm{mod}}} n_{\mathrm{mod}_{i}}-\sqrt{N_{\mathrm{mod}} / N_{\mathrm{obs}}} n_{\mathrm{obs}_{i}}\right)^{2}}{n_{\bmod _{i}}+n_{\mathrm{obs}_{i}}}$

where $n_{\mathrm{obs}_{i}}\left(N_{\mathrm{obs}}\right)$ and $n_{\bmod _{i}}\left(N_{\mathrm{mod}}\right)$ are the number of stars in the $i$ th bin of the $J-K_{\mathrm{s}}$ histogram (total number of stars along the line of sight) of the observations and model, respectively. The term involving the total number of stars ensures that the
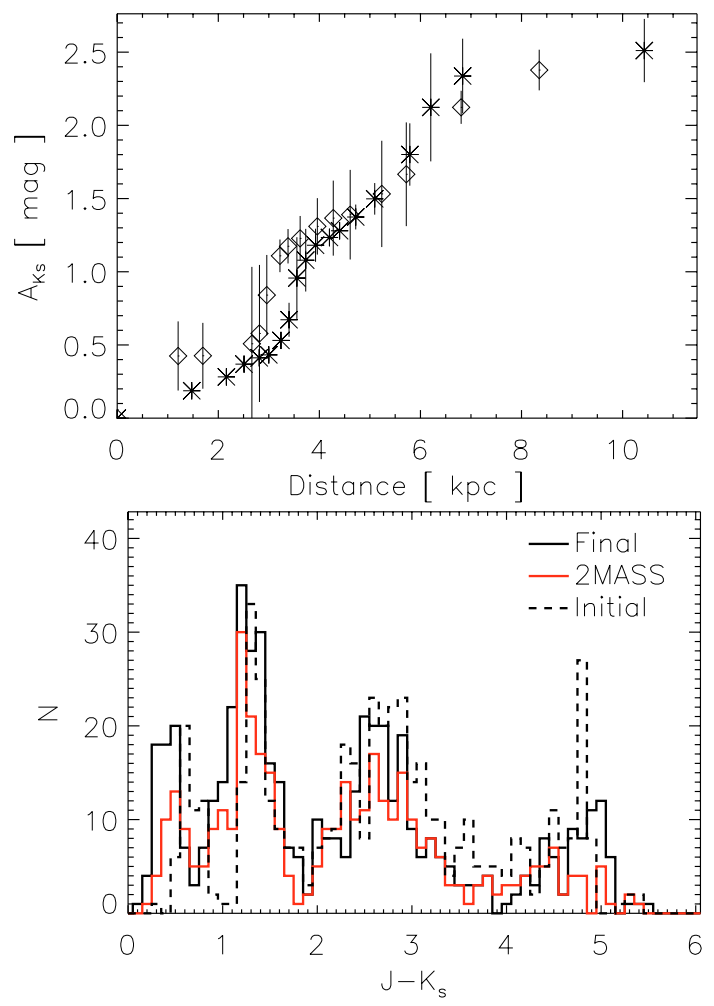

Fig. 4. Initial and final results of the extinction calculation for the field $(l, b)=(345,0) . T o p: A_{K \mathrm{~s}}$ as a function of distance. Each diamond represents a bin from the initial calculation, the asterisks represent the bins in the final result and the error bars represent the mean absolute deviation of the extinction in the bin. Bottom: $J-K_{\mathrm{s}}$ colour distribution of 2MASS and adjusted Galactic model. The red solid line represents the 2MASS data, the bold solid line represents the final result and the broken line shows our initial calculation.

$\chi^{2}$ statistic will be lowest when the shapes of the two histograms are the same, regardless of any difference in the total number of stars.

After having adjusted the extinction some stars will be beyond the completeness limit for the field whereas others, intrinsically bright, will be dim enough to be admitted into our selection. We therefore repeat the process using the adjusted stars and recompute the $\chi^{2}$ statistic. We continue this iterative process until we find a minimum in the $\chi^{2}$ statistic; the line of sight extinction giving rise to this value is then taken to be the best fit for the particular line of sight. This is usually accomplished in under 10 iterations.

This method supposes that the observed and simulated stars in a given bin are at similar distances. As we start with a simple distribution for the extinction this may not be the case, initially. However, each successive iteration improves the extinction estimation and decreases the difference between the observed and modelled colour distributions. Assuming that the spatial density of the giants is well modelled in the Galactic model, and that the extinction is uniform across the field (Sect. 6.3.2), the assumption that the modelled and observed colour bins represent stars at similar distances is justifiable.

As an example, the initial (after one iteration) and final results for a sample field are displayed in Fig. 4. The initial extinction (diamonds) is significantly different from the final extinction (asterisks), with nearly a $1 \mathrm{kpc}$ difference in the location of the sharp rise in extinction at $\sim 3 \mathrm{kpc}$. This difference results 

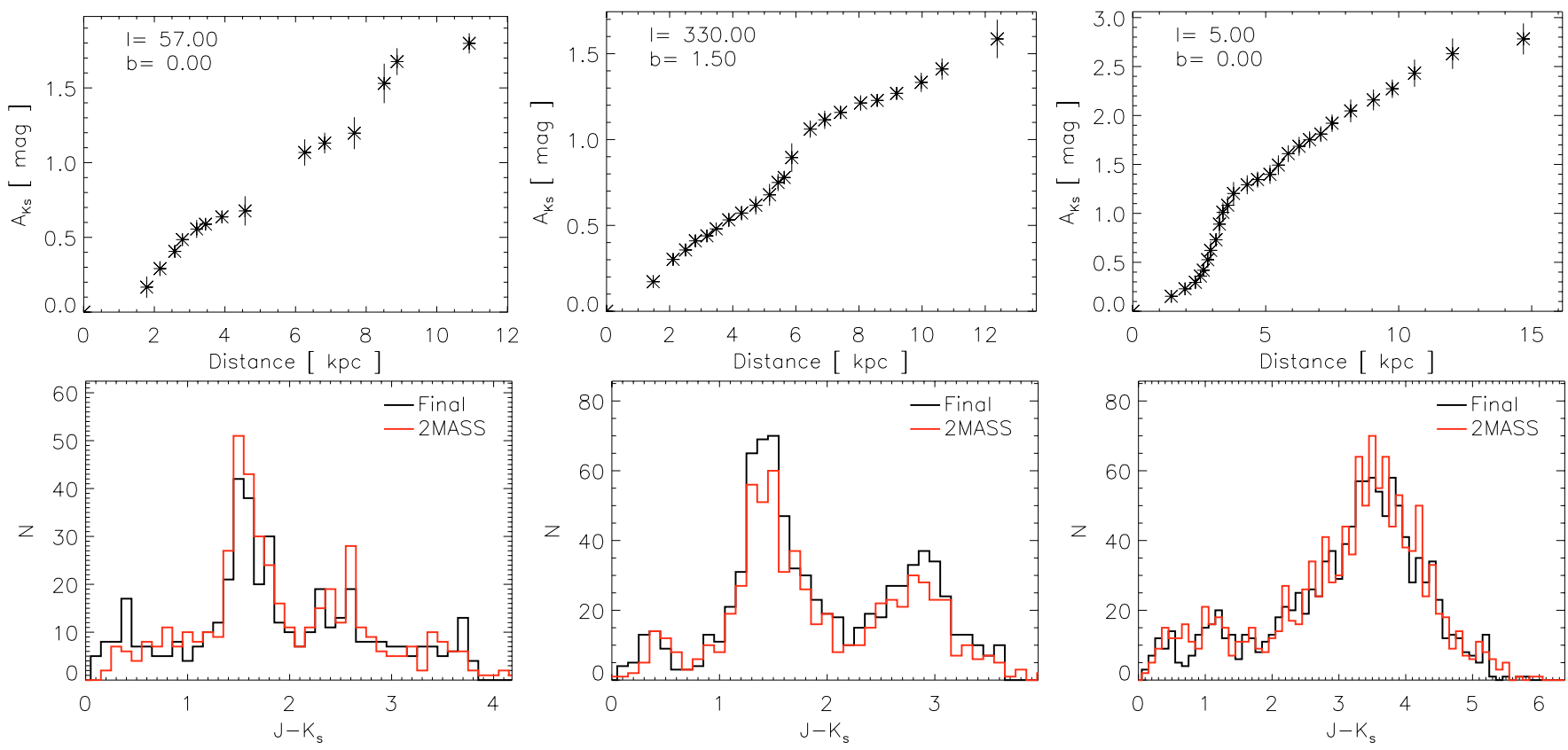

Fig. 5. Results for the three fields at galactic coordinates $(l, b)=(57,0),(330,1.5)$ and $(5,0)$. Top: $A_{K \mathrm{~s}}$ vs. distance. Each asterisk represents a bin from our method, and the error bars are the mean absolute deviation from the median extinction in the bin. Bottom: corresponding $J-K_{\mathrm{S}}$ histogram of the model and the observations. The red line represents the 2MASS observations and the black line is the result of our method.

in a poorer fit to the observed colour distribution and therefore a large value for the $\chi^{2}$ statistic (Eq. (7)).

We may summarise the various steps of the method as follows:

1. assume simple distribution for the extinction (Sect. 3.3), or apply extinction from last iteration;

2. remove dwarf stars and cut observations and model at magnitude limits;

3. sort both catalogues by increasing $J-K_{\mathrm{s}}$ colour;

4. group simulated stars into colour bins such that the distance of each bin $\geq$ distance of previous bin;

5. group observations into same number of bins as simulated star bins, each bin having the same relative number of stars;

6. compute extinction correction using Eq. (5), bin by bin;

7. compute $J-K_{\mathrm{S}}$ histogram and $\chi^{2}$ statistic;

8. continue until the $\chi^{2}$ statistic reaches a minimum.

\section{Results}

The full results are listed in Table 1, only available in electronic form at the CDS. Each row of Table 1 contains the information for one line of sight: Galactic coordinates along with the measured quantities for each bin $\left(A_{K \mathrm{~s}}\right.$, distance and respective uncertainties). All rows also contain the corresponding $\chi^{2}$ statistic to indicate the quality of the fit between the modelled and observed $J-K_{\mathrm{s}}$ histograms.

\subsection{Results for individual lines of sight}

The results for three example lines of sight are presented in Fig. 5: $(l, b)=(57,0),(330,1.5)$ and $(5,0)$. The top row presents the distribution of extinction along the line of sight; each asterisk represents one bin from our results and the error bar is the absolute mean deviation of the $A_{K \mathrm{~s}}$ from the median of all the modelled stars in the bin. Each simulated star at a given distance is attributed an extinction by performing a linear interpolation between the two neighbouring bins. In order to test the extinction law thus determined, we compare the $J-K_{\mathrm{s}}$ histogram from the 2MASS observations to the $J-K_{\mathrm{s}}$ histogram resulting from the application of our extinction to the Galactic model. The histogram for each line of sight is presented in the bottom row of Fig. 5. As can be seen from these three examples, our method works very well in areas of high extinction.

\subsection{Two dimensional extinction maps}

By combining many lines of sight we are able to construct a three dimensional array of extinction values in the Galaxy. However, the values of $A_{K \mathrm{~s}}$ along each line of sight are not distributed uniformly. If we want to know the extinction along a plane at a certain heliocentric distance we must first interpolate in between the values in order to get a regular grid. In what follows we have used linear interpolation between successive bins.

\subsubsection{Comparison with two dimensional extinction maps}

Two dimensional extinction maps towards the Galactic centre were computed by Schultheis et al. (1999) $\left(|l| \leq 8^{\circ},|b| \leq 1.5^{\circ}\right)$ and Dutra et al. (2003) $\left(|l| \leq 5^{\circ},|b| \leq 5^{\circ}\right)$, hereafter S99 and D03 respectively. The method of S99 is based on the comparison of AGB isochrones to theoretical unreddened isochrones (Bertelli et al. 1994), using DENIS data, whereas D03 compute the extinction by fitting the upper giant branch of $\left(K_{\mathrm{s}}, J-K_{\mathrm{s}}\right)$ colour magnitude diagrams to a de-reddened upper giant branch mean locus of previously studied bulge fields.

Although they used near-infrared data as we have, our approach provides a different method for extracting the extinction information from the data.

In order to compare our map with theirs, we first smooth their maps to our resolution of $15^{\prime} \times 15^{\prime}$. The $\mathrm{S} 99$ method yields results in $A_{K \mathrm{~s}}$ but they were published in $A_{V}$; we use the extinction law from Glass (1999), $A_{K \mathrm{~s}}=0.089 A_{V}$, as they did to reconvert the $A_{V}$ to $A_{K \mathrm{~s}}$. The D03 map was published in $A_{K}$ so we 

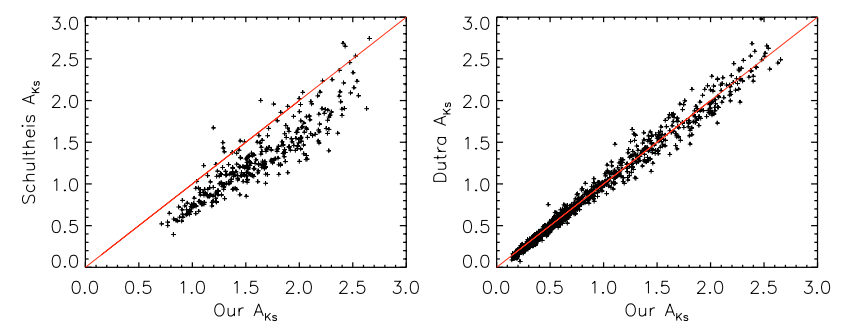

Fig. 6. Comparison between each pixel of our extinction map and that of Schultheis et al. (1999) (S99), for $|l|<8^{\circ},|b|<1^{\circ}$, and Dutra et al. (2003) (D03), for $|l| \leq 5.0^{\circ},|b| \leq 5.0^{\circ}$. Left: $A_{K \mathrm{~s}}$ of our map vs. that of S99. Right: $A_{K \mathrm{~s}}$ of our map vs. that of D03. For both figures, the straight line represents the identity function.

convert it to $A_{K \mathrm{~s}}$ using the conversion factor used in their article $\left(A_{K}=0.95 A_{K \mathrm{~s}}\right)$. Finally, we integrate our extinction to the centre of the Galaxy, assumed to be at $8.5 \mathrm{kpc}$ from the Sun.

The results of this comparison are displayed in Fig. 6, which shows a comparison between our results and the S99 map (left) and D03 map (right) at each pixel.

Although the large scale structure of all three maps are very similar there is a systematic difference of $\sim 0.2-0.3 A_{K \mathrm{~s}}$ between our map and that of S99. This is not surprising as they use the extinction law from Glass (1999), while we use the results of Mathis (1990), resulting in a different ratio for the $K_{\mathrm{s}}$ band to $J$ band extinction $\left(A_{K s} / A_{J}\right)$. This ratio is used in the derivation of Eq. (5) and therefore has a direct impact on the extinction determination.

The extinction law in the infrared can differ significantly from one author to the next; the value we have adopted has been chosen so as to be consistent with the extinction law used for the Galactic model (Mathis 1990).

By combining the Mathis (1990) law, $A_{K} / A_{J}=0.382$ with the relation $A_{K}=0.95 A_{K \mathrm{~s}}$ (Dutra et al. 2002) we obtain $A_{K \mathrm{~s}} / A_{J}=0.402$, very close to the recent value $\left(A_{K \mathrm{~s}} / A_{J}=0.400\right)$ from Indebetouw et al. (2005). However, this value is significantly different from Glass (1999) $\left(A_{K \mathrm{~s}} / A_{J}=0.347\right)$, which is closer to the value recently measured by Nishiyama et al. (2006), $A_{K \mathrm{~s}} / A_{J}=0.331$, in their study of the Galactic bulge.

The application of the Mathis (1990) law to the S99 results effectively removes the systematic shift seen in Fig. 6. D03 already use the same value for $A_{K s} / A_{J}$ as we do; the correlation between our map and theirs is very good. The slight departure from the identity function at low extinction, comes from the poor sensitivity of the $J-K_{\mathrm{s}}$ colour index at these relatively low extinctions (Sect. 6.4.1). These low extinctions do not exist in the S99 map as the area covered by their map does not rise much above $|b| \sim 1.5$; in addition to the more central regions of the Galaxy, the D03 map also probes higher latitude, lower extinction lines of sight.

\subsection{Comparison with velocity integrated CO emission}

Dame et al. (2001) have created a large scale CO survey of the entire Milky Way, using the rotational transition 1-0 of the $\mathrm{CO}$ molecule at $115 \mathrm{GHz}$, from new observations and existing $\mathrm{CO}$ surveys. By comparing the low velocity (local) $\mathrm{CO}$ emission with an optical panorama of the inner Galaxy, they conclude that there are few dark interstellar clouds where $\mathrm{CO}$ is not present or where the temperature is so cold that the $\mathrm{CO}$ molecule fails to radiate at $115 \mathrm{GHz}$. Galactic $\mathrm{CO}$ emission traces only dense regions of the ISM whereas our map also traces the diffuse component. Nevertheless we expect that both maps should trace the large scale distribution of interstellar matter in our Galaxy.

Figure 7 shows the total extinction detected for each line of sight using our method as well as the distribution of $\mathrm{CO}$ as presented in Dame et al. (2001), where the two maps overlap. The resolution of the extinction map is $15^{\prime} \times 15^{\prime}$; the resolution of the $\mathrm{CO}$ map varies from $9^{\prime}$ to $30^{\prime}$, the region that we present here is mostly at the higher resolution of $9^{\prime}$. The main structures that appear in both maps are indicated. The Musca Dark cloud, $(l, b)=(-59,-9)$, that appears in our map is not in the Dame et al. (2001) map as this region was not observed in their composite survey.

\subsection{Three dimensional extinction}

In order to visualize the distribution of extinction elements in three dimensions, we divide the extinction between subsequent bins by the distance between them. The resulting map, in units of $\delta A_{K} \mathrm{kpc}^{-1}$, gives us a better idea of the location of obscuring dust along the line of sight. We assume a constant $\delta A_{K} \mathrm{kpc}^{-1}$ in between bins, as we have no information on the distribution of dust between bins. This results in an overestimation of the line of sight length of clouds for lines of sight with large distances between bins.

These maps are presented in two forms: maps of the full area covered so far by our method at various distances (Fig. 8, and fully described in Sect. 5.4.1), as well as a view of the Galactic plane from above (Fig. 9, referred to in Sect. 5.4.2).

\subsubsection{Distance slices}

The three dimensional extinction towards the inner Galaxy is presented in Fig. 8. Each image shows the average local extinction at distance intervals of $1 \mathrm{kpc}$. The scale is the same for each image and runs from 0 to $0.3 A_{K \mathrm{~s}} \mathrm{kpc}^{-1}$. The solid line superimposed at each distance shows the stellar warp as implemented in the Galactic model.

One obvious feature in the images is the geometrical effect of distance; high latitude extinction is mostly local so the disc is seen to shrink as we go further away from the Sun. In the first couple of slices, the local features mentioned earlier are visible: the Aquila Rift $\left(30^{\circ}, 4^{\circ}\right)$ and the Local Arm towards Cygnus X $\left(80^{\circ}, 0^{\circ}\right)$.

The giant molecular cloud (GMC), and supernova remnant, $\mathrm{W} 44$ is visible in the second and third slices $(2 \mathrm{kpc} \& 3 \mathrm{kpc})$ at $\left(35^{\circ}, 0^{\circ}\right)$. At distances between 3 and $5 \mathrm{kpc}$ the tangent to the Carina arm can be seen at $\sim-78^{\circ}$.

After $6 \mathrm{kpc}$, a lack of absorbing material is noticeable towards the Galactic bulge, an effect noted by Glass et al. (1987) who found that the bulge has little dust compared to its CO emission. At these distances another feature of the disc which starts to appear is the Galactic warp. It becomes more pronounced at large absolute longitudes as we move farther out from the Sun.

Finally, the lack of line of sight resolution for certain fields is obvious at $(l, b) \sim(30,5)$ and at distances greater than $3 \mathrm{kpc}$. Due to the fact that we use linear interpolation between successive bins and therefore do not assume any structure for the distribution of the dust in between bins, the large distance between successive bins creates an unrealistically long structure along certain lines of sight. The extinction estimation itself is still assumed to be correct, within error. 


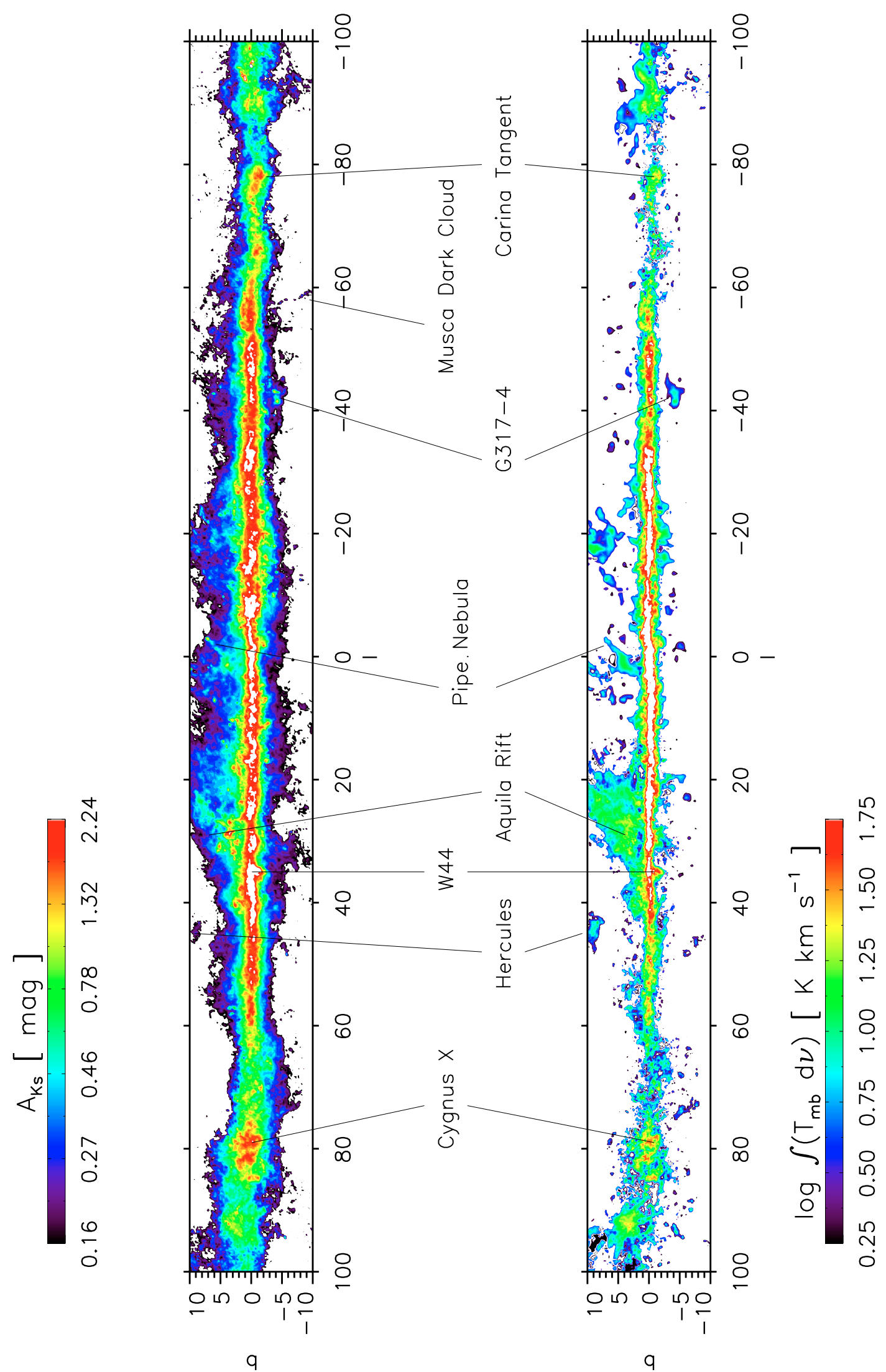

Fig. 7. Top: total extinction integrated along the line of sight. Bottom: CO velocity integrated spatial map by Dame et al. (2001). The units of the two maps are different; they are put here to compare their respective interpretation of the large scale structure of the Galaxy. The coordinates are expressed in degrees $(l, b)$. 

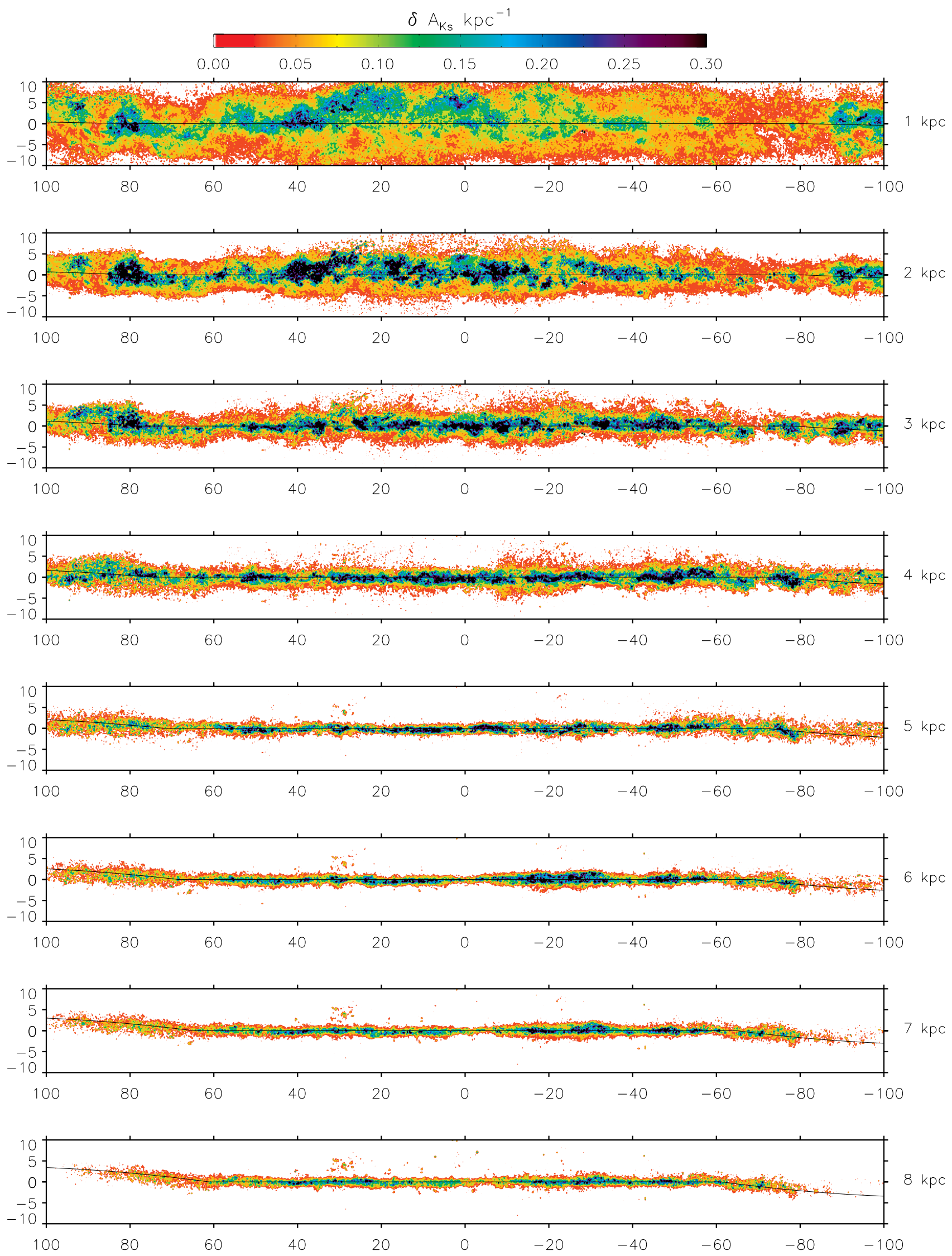

Fig. 8. The local extinction at $1 \mathrm{kpc}$ intervals from the sun. The uppermost image is at $1 \mathrm{kpc}$, the bottom one is at $8 \mathrm{kpc}$. The $x$ axis is in Galactic longitude, the $y$ axis in Galactic latitude. The solid line indicates the mean position of the plane, as given by the stellar warp formula in the Galactic model. Different structures are identified in the text (Sect. 5.4.1). 


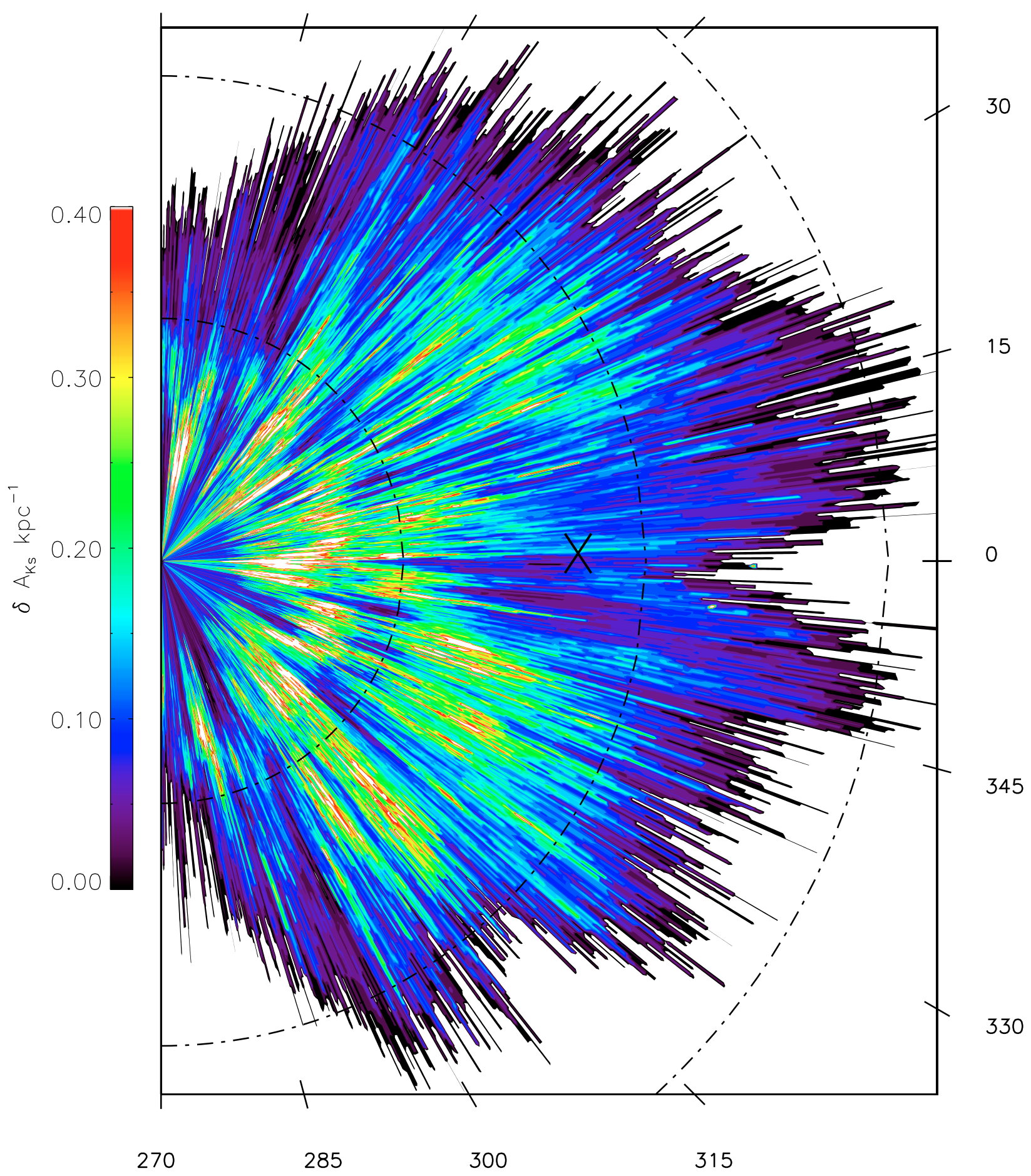

Fig. 9. Location of absorbing dust in the Galactic Plane $(|b| \leq 0.25)$. The Galactic centre, assumed to be at $8.5 \mathrm{kpc}$ from the Sun, is marked with an "X"; the Sun is located in the middle on the left side of the plot. The dotted lines are placed every 5 kpc from the Sun. Ticks on the border indicate Galactic longitude.

\subsubsection{The Galactic plane}

By creating a map showing the local extinction in $\delta A_{K \mathrm{~s}} \mathrm{kpc}^{-1}$, we are able to visualise the location of absorbing matter in the inner Galaxy. A view "from above" is displayed in Fig. 9 which is the average $\delta A_{K \mathrm{~s}}$ for $|b| \leq 0.25$ towards the inner Galaxy. The Sun is located at the middle left of the graph, and the Galactic centre is marked by an " $\mathrm{X}$ ".
Many structures are visible in this figure, such as the local arm extending outwards from the Sun at $l \sim 80^{\circ}$. Two tangents to the Sagittarius-Carina arm are also visible, extending from the Sun in two directions at $l \sim 280^{\circ}$ and $l \sim 50^{\circ}$. The outer Perseus arm is above the top edge of the map and is therefore not readily identifiable. The inter-arm region between the Centaurus-Crux and Norma arms is visible at $\sim 4.0 \mathrm{kpc}$ from the Galactic Centre. After the Norma arm, centred on the Galactic centre is a large 


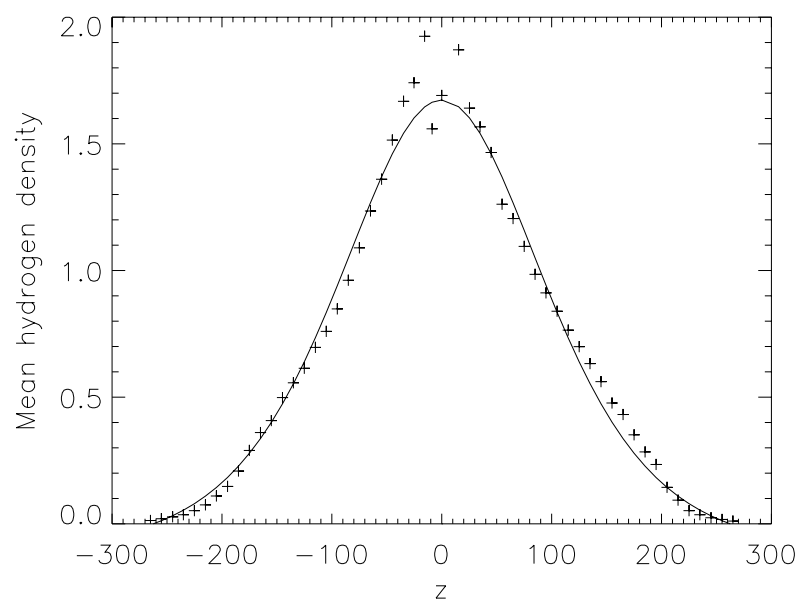

Fig. 10. Variation of the density of absorbing matter as a function of distance from the Galactic plane.

void of dust apart from an elongated structure which may well indicate the presence of dust in the Galactic bar, which we discuss below.

\subsection{Galactic parameters}

We concentrate on determining large scale characteristics of the 3D dust distribution from our 3D extinction map. The mean scale height, as well as the shape of the warp are measured. An estimate of the central dust bar orientation and size is also given.

\subsubsection{ISM disc scale height}

Our 3D extinction map allows us to calculate the scale height of the dust component of the ISM. The extinction between two successive bins was converted to a hydrogen column density using the results from Bohlin et al. (1978); this column density was then divided by the distance between the bins to obtain a hydrogen density per volume. This supposes that the metallicity is the same as in the solar neighbourhood; Chiappini et al. (2001) show that the abundance gradient in the Milky Way is relatively flat between 4 and $10 \mathrm{kpc}$ from the Galactic centre but that it steepens sharply in the outer disc. To minimise the effect of any such gradient on our calculation, we avoid all areas within $4 \mathrm{kpc}$ of the Galactic centre and beyond the solar circle. We then calculated the mean hydrogen density at every $10 \mathrm{pc}$ from the Galactic plane.

In order to measure the scale length of the detected interstellar matter, we tested two profiles:

$\rho=\rho_{0} \operatorname{sech}^{2}\left(z / h_{z}\right)$

$\rho=\rho_{0} \mathrm{e}^{\left(-|z| / h_{z}\right)}$

where $h_{z}$ is the scale height of the ISM and $\rho_{0}$ the hydrogen density in the Galactic plane. The use of the $\operatorname{sech}^{2}$ profile results in a better fit and is shown in Fig. 10. The exponential gives $h_{z}=$ $134_{-11}^{+44}$ pc and $\rho_{0}=2.5 \pm 1.3$ atoms $\mathrm{cm}^{-3}$, whereas the $\mathrm{sech}^{2}$ profile yields $h_{z}=125_{-7}^{+17} \mathrm{pc}$ and $\rho_{0}=1.8 \pm 1.0$ atoms cm $\mathrm{cm}^{-3}$. Both Malhotra (1995) and Nakanishi \& Sofue (2003) find that the scale height of the HI disc in the Milky Way varies as a function of distance; for both the mean value between 4 and $8 \mathrm{kpc}$ is of the order of $\sim 160 \mathrm{pc}$. The dust model of Drimmel \& Spergel (2001), adjusted to the FIR emission, includes a scale height of $134.4 \pm 8.5 \mathrm{pc}$. We were not able to detect any significant flaring
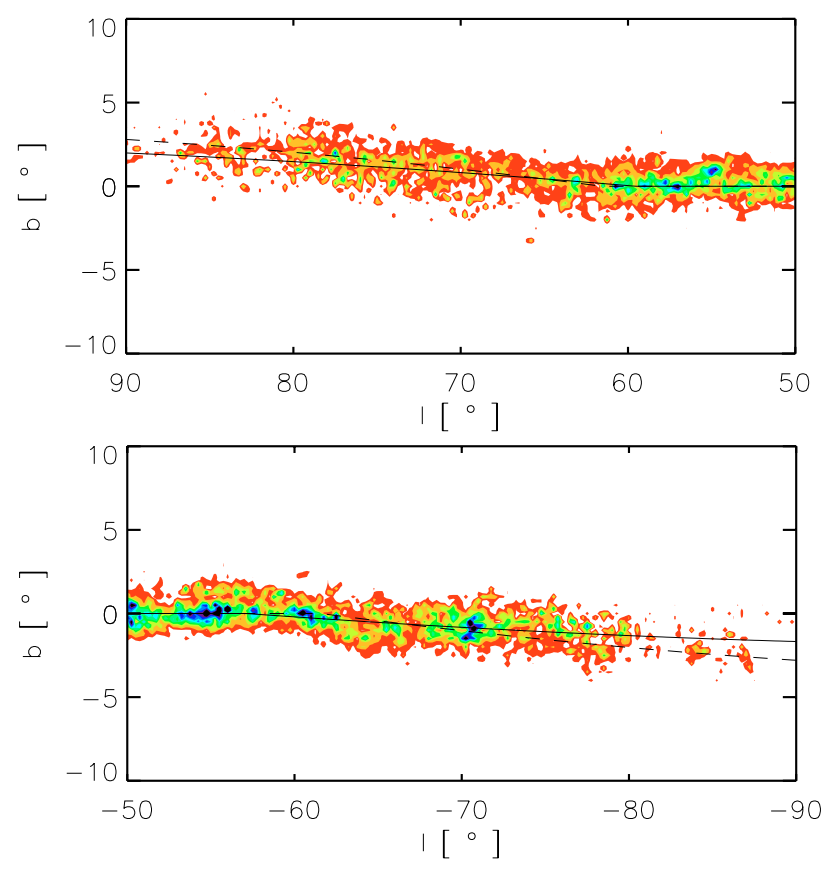

Fig. 11. Local extinction at $8 \mathrm{kpc}$ from the Sun for positive longitudes (top) and negative longitudes (bottom). The stellar warp as modelled in the Galactic model is shown as a dashed line; the solid line shows the best fit to our extinction data (Sect. 5.5.2). The colour scale is the same as in Fig. 8.

of the interstellar matter disc within the Galactocentric radii we tested.

\subsubsection{Galactic warp}

The Galactic warp is obvious in Fig. 8, especially at large absolute longitudes and heliocentric distance. The magnitude of the warp in the Galactic model is calculated using Eq. (10):

$z_{\mathrm{warp}}=\gamma *\left(R-r_{\mathrm{warp}}\right) * \cos \left(\theta-\theta_{\text {warp }}\right)$

where $z_{\text {warp }}$ is the perpendicular shift from the mean $b=$ $0^{\circ}$ plane, $R, \theta$ and $z$ are Galactocentric coordinates, $\gamma$ is the slope of the displacement of the mid plane with respect to the plane of symmetry, $r_{\text {warp }}$ is the radius after which the warp starts and $\theta_{\text {warp }}$ is the angle of maximum warp. Using our results, we are able to locate the $z$ with the highest density for each $(r, \theta)$ by calculating the best fit with Eq. (10). We find that the warp is not symmetrical; for positive longitudes we find $\gamma=0.14, \theta_{\text {warp }}=89^{\circ}$ and $r_{\text {warp }}=8.7 \mathrm{kpc}$ and for negative longitudes we find $\gamma=0.11$, $\theta_{\text {warp }}=272^{\circ}$ and $r_{\text {warp }}=7.8 \mathrm{kpc}$. Figure 11 shows the local extinction per kpc at a heliocentric distance of $8 \mathrm{kpc}$, overlaid with the warp parameters of the Galactic model (dashed line) and with the above parameters (solid line). These values correspond to an absolute vertical shift of the plane at $R=12 \mathrm{kpc}$ of $460 \mathrm{pc}$ at both negative and positive longitudes, significantly smaller than the modelled stellar warp which predicts a vertical shift of $\sim 650 \mathrm{pc}$ at $R=12 \mathrm{kpc}$. These new values for the dust warp are slightly higher than those of Drimmel \& Spergel (2001) $\left(\gamma=0.0728, r_{\text {warp }}=6.993 \mathrm{kpc}\right)$, who predict a maximum vertical shift of $364 \mathrm{pc}$ at $R=12 \mathrm{kpc}$. This shift is different for the stellar warp, which they find to be higher. López-Corredoira et al. (2002) use a different formulation to model the warp and find the maximum vertical shift of the stellar warp at $R=12 \mathrm{kpc}$ to be $547 \mathrm{pc}$, in good agreement with the gas warp found by Burton (1988). 


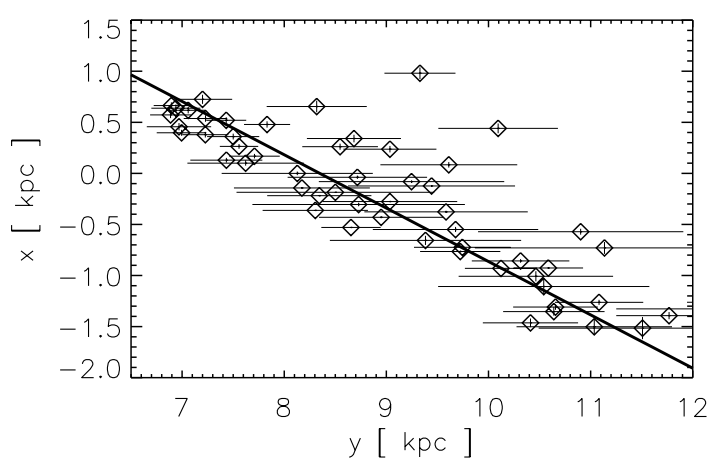

Fig. 12. Straight line fit that minimises the mean absolute deviation of the points of highest density along the Galactic bar.

Nakanishi \& Sofue (2003) find that the Galactic HI disc is warped and asymmetric, with a larger shift at positive longitudes, as we do. They report that the warp is at a maximum for $\theta=80^{\circ}$ and $\theta=260^{\circ}$, and that the warp starts at $R=12 \mathrm{kpc}$, contrary to our values at around $R=8 \mathrm{kpc}$. At positive longitudes the warp continues to reach a maximum displacement from the $b=0^{\circ}$ plane of $1.5 \mathrm{kpc}$ at $R=16 \mathrm{kpc}$, resulting in a slope of 0.375 . At negative longitudes they find that the warp is less severe, resulting in a shift of $1.0 \mathrm{kpc}$ below the $b=0^{\circ}$ plane at $R=16 \mathrm{kpc}$, a slope of 0.25 . Their values for the gradient of the warp are significantly higher than ours, however our map does not have information out to these large galactocentric distances and so we are not able to constrain the large scale attributes of the interstellar warp in the outer Galaxy.

Most recently Levine et al. (2006) have shown that the outer HI warp can be described by a superposition of the three low mode ( $m=0,1$ and 2 ) vertical harmonics of the disc, one of which ( $m=1$ corresponding to the integral-sign shape) dominates the warp for $R \lesssim 15 \mathrm{kpc}$. It seems that it is this mode that we see in the dust from our study, which does not extend far enough into the external regions of the Galaxy to detect the other modes. They find that this mode is approximately linear for $10 \lesssim R \lesssim 15 \mathrm{kpc}$ and that it has a slope of 0.197 , significantly higher than our value.

From these comparisons one sees that the warp is present in all galactic components (dust, gas and stars), all with the same node position and all asymmetric. However, the amplitude of the warp seems to depend slightly on the component one looks at: the dust warp (our study; Drimmel \& Spergel 2001) seems to be less pronounced that the stellar warp (the Galactic model; López-Corredoira et al. 2002; Drimmel \& Spergel 2001), itself less pronounced than the HI warp (Nakanishi \& Sofue 2003; Levine et al. 2006). However, this conclusion has to be taken with caution because the error bars are still large and our study does not go far enough into the external Galaxy.

\subsubsection{Dust bar}

The visualisation of our extinction map in the plane Fig. 9 reveals an elongated structure passing through the Galactic centre. To determine its parameters, we fit the straight line to this bar that minimises the mean absolute deviation of the points of highest density along the bar (Fig. 12).

We find that the dust bar is at an angle of $\phi=30 \pm 5^{\circ}$ relative to the Sun - Galactic centre direction. The structure thus defined has a length of $5.2 \mathrm{kpc}$. The angle found is higher than the modelled stellar bar (Sect. 3.2.2) and that found by Babusiaux \& Gilmore (2005) $\left(\phi=22.5 \pm 5.5^{\circ}\right)$, but still within the uncertainty of the latter. Furthermore it is compatible with the values cited in Gerhard (2001), who find that the angle may lie between $\phi=15^{\circ}$ and $\phi=35^{\circ}$, although the bar angles based on stellar studies are systematically lower than the value we have found. Bissantz \& Gerhard (2002) find that COBE/DIRBE observations are compatible with an angle for the stellar bar between $\phi=15^{\circ}$ and $\phi=30^{\circ}$, but that the model with an angle of $\phi=20^{\circ}$ fits the observations best. These results for the stellar bar suggest that the dust bar we have detected may in fact be dust lanes which precede the stellar bar at negative longitudes and trail it at positive longitudes, a feature of our Galaxy suggested by Calbet et al. (1996). However, we detect a difference in extinction between positive and negative longitudes of $\sim 0.1 A_{K \mathrm{~s}}$, much smaller than their value of between 1 and $2.6 A_{K}$.

\subsection{Comparison with other $3 D$ methods}

As detailed in Sect. 1, other methods have been developed to measure interstellar extinction along the line of sight. We now present our results alongside those of Neckel et al. (1980), López-Corredoira et al. (2002) and Drimmel et al. (2003).

The three methods are described briefly below; for further information, please refer to the corresponding article.

Neckel et al. (1980): extinction values and distances are given for over $11000 \mathrm{O}-\mathrm{F}$ stars. The intrinsic colour and absolute magnitude of the stars are deduced from the MK spectral type and from the $\mathrm{H} \beta$ index using the relationship between $M_{V}$ and $\beta$.

López-Corredoira et al. (2002): this method is based on 2MASS observations of red clump giants, for which a value of the absolute magnitude and $J-K_{\mathrm{S}}$ colour is assumed $\left(M_{K_{\mathrm{s}}}=-1.65\right.$ and $\left.\left(J-K_{\mathrm{s}}\right)_{0}=0.75\right) .\left(J-K_{\mathrm{s}}, M_{K_{\mathrm{s}}}\right)$ CMDs of the giants are built for $1^{\circ} \times 1^{\circ}$ fields and their displacement from an unreddened CMD, along with their absolute magnitude, are used to calculate the extinction and thus the distance to the extinction.

Drimmel et al. (2003): the three dimensional dust model by Drimmel \& Spergel (2001) is used to calculate the three dimensional extinction. The dust model is adjusted using the far-infrared residuals between the DIRBE $240 \mu \mathrm{m}$ data and the emission predicted from the model.

Results for various lines of sight are presented in Fig. 13. The Neckel et al. (1980) data is the one with the largest dispersion: young and hot OB stars are often surrounded by their parent cloud and are thus subject to possibly large and varying circumstellar extinction and therefore not representative of the whole field. Our results follow the López-Corredoira et al. (2002) results quite closely; this is to be expected as they are both based on the near infrared colour difference of giant stars. However significant differences do exist along certain lines of sight; this may be due to the fact that they do not use a model to determine the distance but instead use a unique absolute magnitude for all stars. Furthermore they do not use the same stellar populations as us, isolating the $\mathrm{K}$ giants from dwarfs and $\mathrm{M}$ giants using the "SKY" model (Wainscoat et al. 1992). In areas where this single population hypothesis doesn't hold differences between our results would be expected.

Finally, we are not always in agreement with Drimmel et al. (2003). This is also to be expected given the region under study; anomalous dust temperatures, as well as lines of sight passing through spiral arm tangents or near the Galactic centre may introduce systematic errors in the Drimmel et al. (2003) extinction 

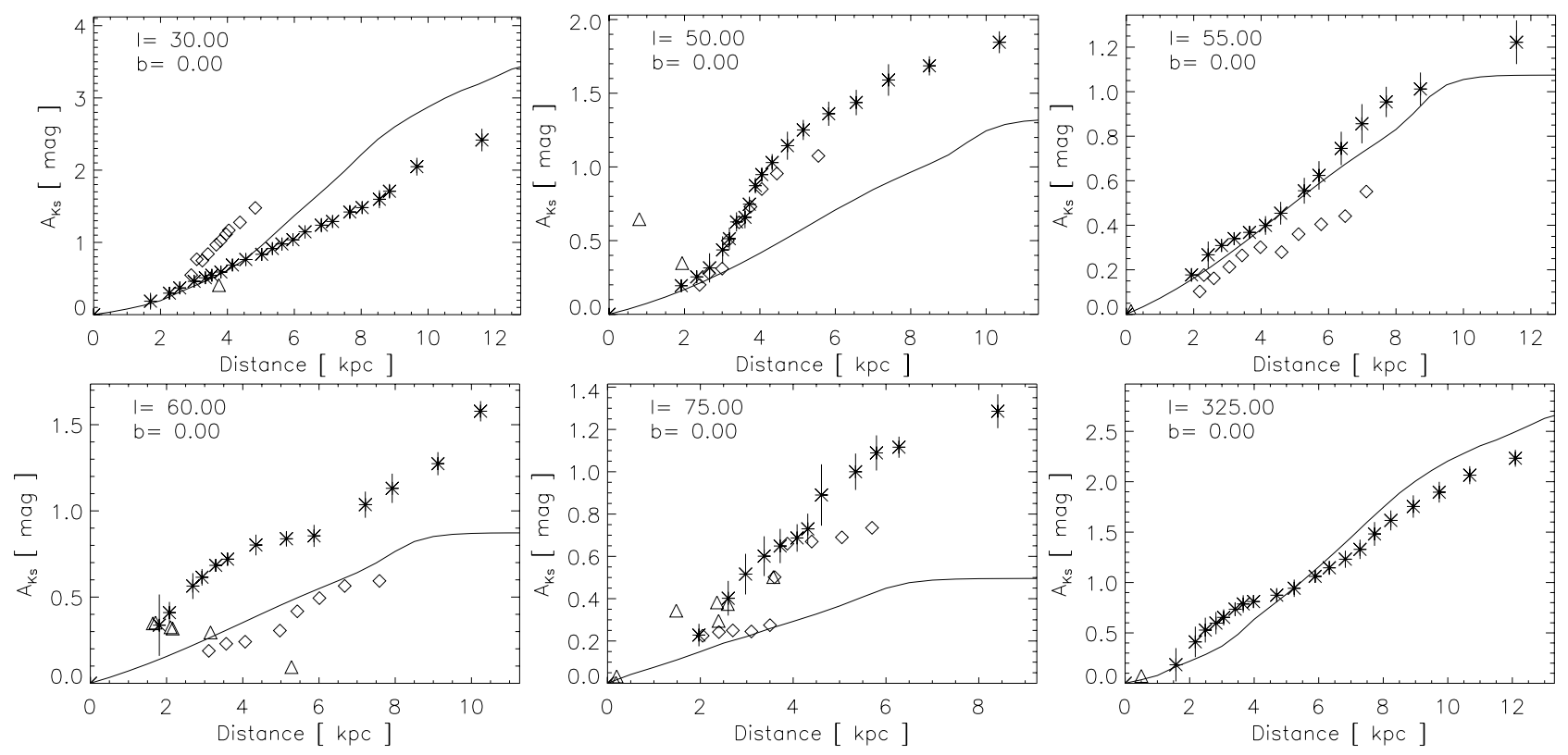

Fig. 13. The extinction, along the line of sight, in six different fields. For each field, we have compared our results (asterisks) with the results of Neckel et al. (1980) (triangles), Drimmel et al. (2003) (solid line) and López-Corredoira et al. (2002) (diamonds), where available.

map. Contrary to theirs, our map is not sensitive to dust temperature or to an assumed spiral structure but to the modelled K\&M giant luminosity function (see Sect. 6.2.2).

\section{Discussion}

\subsection{Use of the extinction maps}

Our results are available in the form of an electronic three dimensional map. The map is presented in $A_{K s}$, as the extinction law in this wavelength range is nearly universal (Mathis 1990) and so does not depend on the ratio of total to selective extinction. By adopting a suitable extinction law (for example Mathis 1990; Rieke \& Lebofsky 1985 or Glass 1999), one can convert this $K_{\mathrm{s}}$ band extinction to an extinction in the visible portion of the spectrum or any other.

It was not possible to apply our method to some lines of sight in the region we have studied $\left(|l| \leq 100^{\circ},|b| \leq 10^{\circ}\right)$ either because there were an insufficient number of stars in the 2MASS observations for our pixel size or the extinction was below the sensitivity of our method (Sect. 6.4.1). In these cases we have substituted the results of our method with the diffuse extinction disc of the Galactic model (Sect. 3.3), with a local normalisation chosen to minimise the $\chi^{2}$ test described in Sect. 4.4. This solution was also preferred in regions where our method could not improve on the extinction predicted by the default extinction of the Galactic model. To aid the user of our map, we have included the $\chi^{2}$ statistic for each line of sight.

Our results are most reliable in fields with homogeneous extinction. As this is not always the case, special care should be taken when using fields with large values for the $\chi^{2}$ statistic. In these cases, our method was not able to find a satisfactory solution for the line of sight extinction.

\subsection{Effect of changing the model parameters}

The determination of extinction using the Galactic model can be affected by changing the model parameters. The magnitude of the extinction that we calculate depends on the difference in colour between observed and modelled stars; the distance to the modelled stars (and hence to the extinction) is mostly dependent on the assumed absolute magnitude of the dominant population.

This feature of our method means that the number of stars does not affect the extinction determination, so that if there are a number of stars in the model which are not present in the observations, we do not "invent" extinction in order to remove the excess stars from the model.

The performance of the Galactic model to reproduce observations in low extinction windows is a useful measure of the validity of the model. This comparison was done in Robin et al. (2003); star count predictions (stars per magnitude and per square degree) in the $K_{\mathrm{s}}$ band were in good agreement with observations from the DENIS survey and so we refer the interested reader to the aforementioned publication.

We now briefly describe the effect on the extinction determination of: the size of the hole in the modelled disc distribution (Sect. 6.2.1); changing the bulge luminosity function (Sect. 6.2.2); and changing the stellar density (Sect. 6.2.3) on the resulting extinction distribution.

\subsubsection{Galactic disc hole}

In Fig. 14 we show the median difference between each pixel of two extinction maps (map1 minus map2), at different heliocentric distances and for $|l| \leq 10^{\circ}$ and $|b| \leq 2.5^{\circ}$. The Galactic model used for map1 has a central hole in the disc population of $1360 \mathrm{pc}\left(R_{\mathrm{h}}=1360 \mathrm{in} \mathrm{Eq.} \mathrm{(1))} \mathrm{and} \mathrm{the} \mathrm{model} \mathrm{used} \mathrm{for} \mathrm{map2}\right.$ includes a hole with a diameter of $680 \mathrm{pc}$. The scale in the figure runs from -0.1 to $0.1 A_{K \mathrm{~s}}$; a positive difference indicating more extinction in the large hole model. As can be seen in Fig. 14, for distances closer than $6 \mathrm{kpc}$ the large hole model predicts more extinction. We use the Galactic model to calculate the distance to the extinction: with fewer "distant" stars in the big hole model, we place the detected extinction closer than for the small hole model. The median difference is never large, however, and stays within the error of the method (Sect. 6.4.1). 


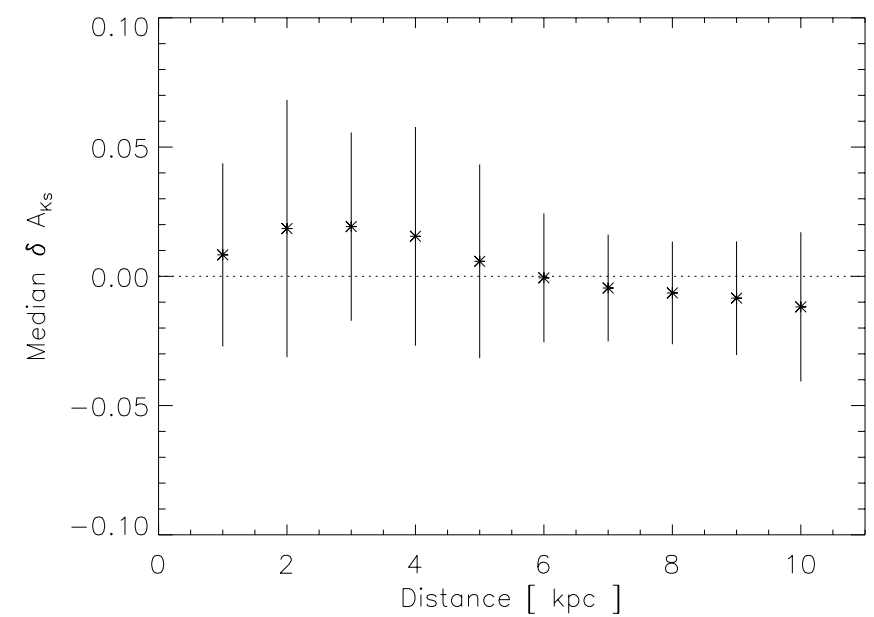

Fig. 14. Influence of the assumed disc density law near the Galactic centre. Median extinction difference, at different heliocentric distances and at $|l| \leq 10^{\circ}$ and $|b| \leq 2.5^{\circ}$, between two determinations of the extinction using models with different values for the disc hole scale length $\left(R_{\mathrm{h}}\right.$ in Eq. (1)). The error bars represent the mean absolute deviation from the median. The difference is calculated per pixel, at each heliocentric distance. A positive difference shows that the model with the larger $R_{\mathrm{h}}$ predicts a larger value for the extinction.

\subsubsection{Bulge luminosity function}

The effect of changing the bulge luminosity function on the determination of the extinction has been tested. Three luminosity functions were used:

Padova 7.9 and 10.0 Gyr Deduced from theoretical isochrones by the Padova team (Girardi et al. 2002), of which we test two bulge ages: $7.9 \mathrm{Gyr}, 10 \mathrm{Gyr}$;

Bruzual 10.0 Gyr taken from the evolutionary bulge synthesis models of Bruzual \& Charlot (Bruzual et al. 1997). We test their 10 Gyr model.

all of them based on a Salpeter initial mass function $(\alpha=2.35)$ and assuming a single epoch of formation (starburst) as well as a mean solar metallicity $(Z \approx 0.02)$.

Although these luminosity functions alter the distribution of extinction along certain lines of sight, the overall effect is minimal. The extinction resulting from the use of each luminosity function was compared at intervals of $0.5 \mathrm{kpc}$ for various lines of sight towards the outer Galactic bulge and the maximum difference in $A_{K \mathrm{~s}}$ between the resulting extinction distributions at each distance point was recorded. The ensuing difference distribution has a mean of $0.021 A_{K \mathrm{~s}}$ and a standard deviation of $0.018 A_{K \mathrm{~s}}$. As this value is comparable to the error of our method (Sect. 6.4.1), we choose to only use the default luminosity function for the Galactic model, namely that of Bruzual 10.0 Gyr.

\subsubsection{Galactic scale length}

When modelling the Galactic disc, the density is normalised at the solar position. Therefore, in the plane of the Galaxy, the stellar density of K\&M giants is largely determined by the scale length of the old disc (Eq. (1)). As the scale length shortens, the number of stars towards the Galactic centre increases, while an increase in the scale length has the opposite effect.

In order to test the robustness of our method to a change in stellar density, we replace the 2MASS observations in our method with simulated observations with a known extinction distribution using the Galactic model. In the following, we vary the Galactic scale length in the simulated observations. We then proceed as normal (Sect. 4), using the default model to determine the extinction. This simulates the impact on our method of choosing a value for the scale length of the Galaxy which is up to $25 \%$ different from the observations.

In Fig. 15 we show the effect of setting the scale length of the simulated observations to $2510 \mathrm{pc}$ (the default value for the Galactic model), $2000 \mathrm{pc}$ and $3000 \mathrm{pc}$. The top row of this figure shows the extinction distribution of the simulated observations (solid line) and the extinction distribution found using our model (asterisks with error bars). The simulated observations have been subjected to a diffuse extinction as described in Sect. 3.3 with a local normalisation of $0.7 \mathrm{mag} \mathrm{kpc}^{-1}$ plus two clouds at 2 and $6 \mathrm{kpc}$. The bottom row shows the $J-K_{\mathrm{s}}$ histogram of the simulated observations and of the default model with the calculated extinction distribution.

The calculated extinction is found to reproduce the simulated observations' extinction very well, and always within the error bars. At large distances a small systematic difference is seen: if we assume a value of $2510 \mathrm{pc}$ for the scale length while setting it to $2000 \mathrm{pc}$ in the simulated observations, we produce an underestimation of the extinction of $\$ 10 \%$ at distances larger than $12 \mathrm{kpc}$. Underestimating the scale length (assuming $2510 \mathrm{pc}$ when it is $3000 \mathrm{pc}$ in the observations) results in a slight overestimation of the extinction at large distances; at shorter distances, the position of the first cloud is also displaced by $0.5 \mathrm{kpc}$.

\subsection{Bias and uncertainty}

\subsubsection{Circumstellar dust}

As we are using $\mathrm{K} \& \mathrm{M}$ giants as a tracer of interstellar extinction, our stellar selection will be contaminated by AGB stars with circumstellar dust, making them redder than can be accounted for by interstellar extinction alone. $M$ giants with spectral type later than M5 also show signs of circumstellar dust, although the amount has been shown to be negligible (Glass \& Schultheis 2003). In most parts of the Galaxy the ratio of AGB stars to RGB stars is small, of the order of a few \%. Only in the inner parts of the Galactic bulge (at $|l|<2$ and $|b|<2$ ) does the fraction of AGB stars become significant (up to $\sim 20 \%$ ). However, number densities of the different types of AGB stars such as semiregular variables, Mira-type variables and the high luminosity $\mathrm{OH} / \mathrm{IR}$ stars are still very poorly known (Habing 1996).

Jura \& Kleinmann (1989) studied the number densities of mass-losing AGB stars in the solar neighbourhood and estimated their surface density at around $\sim 25 \mathrm{kpc}^{-2}$. Le Bertre et al. (2003) used the Japanese space experiment IRTS to investigate the spatial distribution of mass-losing AGB stars. They found that objects with high mass-loss in the order of $10^{-6}<$ $M_{\odot} / \mathrm{yr}<10^{-5}$ dominate the replenishment of the ISM, however these sources constitute a minority of the total number of stars in our sample $(\sim 10 \%)$. These stars have a large near-IR excess in $J-K(>0.5 \mathrm{mag})$.

We conclude that there is only a very small level of contamination by high-mass losing AGB stars in our selection, mainly in the region of the inner bulge. Our values for the extinction in this region may be slightly overestimated at large distances.

\subsubsection{Cloud substructure}

For each line of sight measurement, the extinction that we detect is assumed to cover the entire field at the given resolution. However, Faison \& Goss (2001) have resolved structure in the 

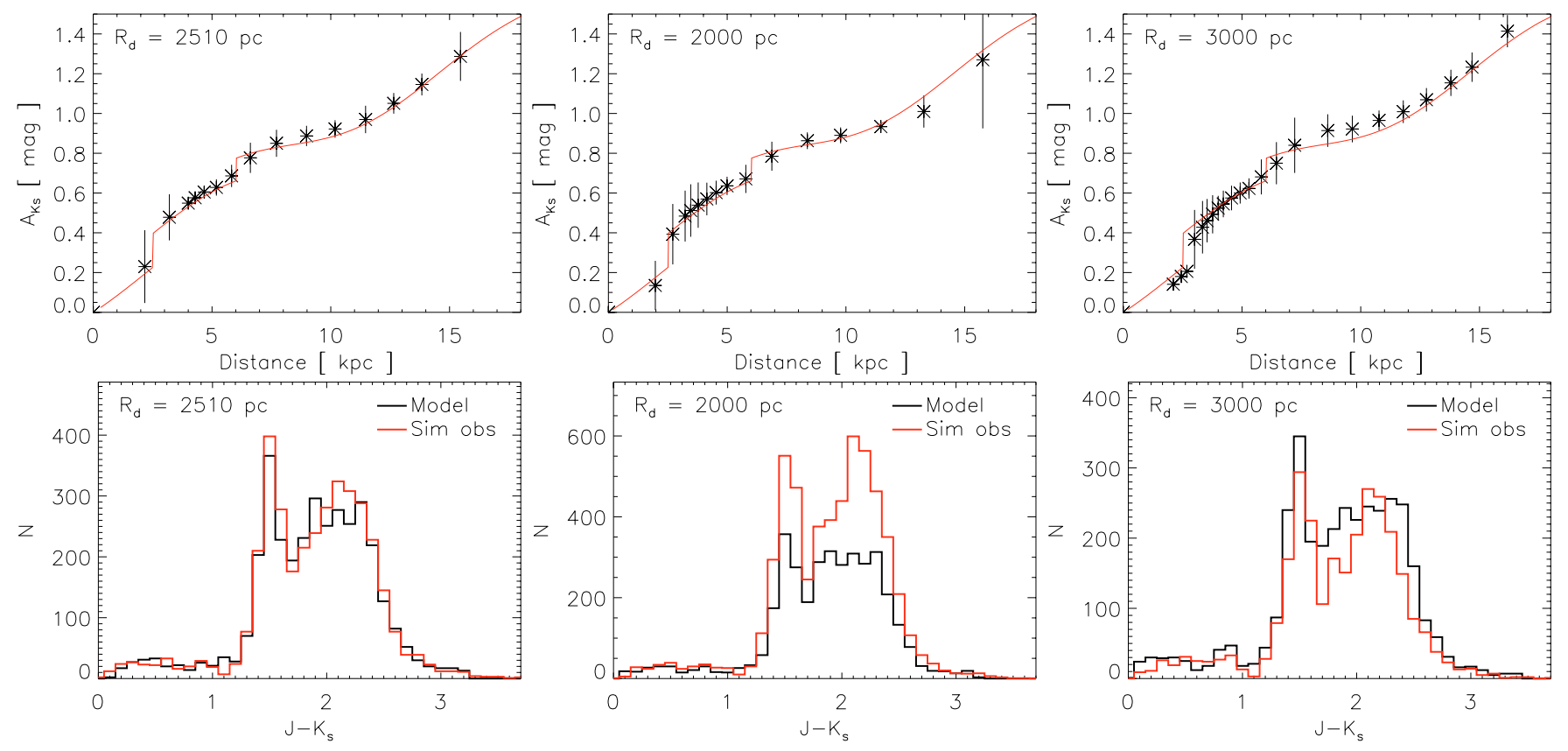

Fig. 15. Influence of the assumed disc scale length. Simulated observations are computed with a scale length of $2.51 \mathrm{kpc}$ (first column), 2. kpc ( $2 \mathrm{nd} \mathrm{col}$. ), and 3. kpc (3rd col.) while the scale length of the model used for computing the extinction is assumed to be $2.51 \mathrm{kpc}$ in each case. Top: the distribution of extinction along the line of sight at $(l, b)=\left(10^{\circ}, 0^{\circ}\right)$. The solid line represents the known extinction law of the simulated observations and the asterisks are the results of our method. In each case the scale length used in the simulated observations is included in the graph. Bottom: the corresponding $J-K_{\mathrm{s}}$ histograms with the extinction applied. It is noticeable that the fact that the model does not reproduce the number density of stars in the observations does not influence the determined extinction.

neutral interstellar medium using the Very Long Baseline Array at scales as small as $10 \mathrm{AU}$. The resolution of our map is 15'; as we cannot resolve structure closer than $\sim 1 \mathrm{kpc}$, we will not resolve any cloud smaller than $\sim 5 \mathrm{pc}$ across.

Nevertheless, as we are placing ourselves in the context of the large scale fluctuations in the ISM, the effects of these small clouds do not have a significant effect on our estimation. Clouds with an angular size much smaller than our pixel size will introduce anomalous reddening for a small fraction of the stars in a given field. Our use of the median as an estimator excludes these outlier points.

\subsection{Limits}

\subsubsection{Maximum and minimum extinction detectable}

At low $A_{K \mathrm{~s}}$, stars that are detected in the $K_{\mathrm{s}}$ band are almost certainly detected in the $J$ band. However, as column density increases and the reddening of the sources gets larger the chances of detecting a source in the $K_{\mathrm{S}}$ band without a corresponding detection in the $J$ band increases.

No selection effect is introduced, however, as we reject the modelled stars in the same way as they are in the data (same limiting magnitude in the $J$ and $K_{\mathrm{s}}$ bands). Instead, this will introduce a maximum $A_{K \mathrm{~s}}$ in the map as very red stars will not be detected.

This upper limit depends on the magnitude limit in the $J$ band of the 2MASS observations. As we place a lower bound on the $K_{\mathrm{s}}$ magnitude of 9 , the highest $J-K_{\mathrm{S}}$ colour index observable along a particular line of sight would be $J_{\text {comp }}-K_{\mathrm{s}(\min )}$ where the subscript comp signifies the completeness limit and min the brightest magnitude used due to our self imposed limit. The highest extinction observable along a line of sight will then be:

$$
A_{K \mathrm{~s}(\max )}=0.67 \times\left[\left(J_{\mathrm{comp}}-K_{\mathrm{s}(\min )}\right)-\left(J-K_{\mathrm{s}}\right)_{0}\right]
$$

where $\left(J-K_{\mathrm{S}}\right)_{0}$ is the intrinsic $J-K_{\mathrm{S}}$ colour of a $\mathrm{K}$ or M giant. If we suppose that for a K2 Giant $\left(J-K_{\mathrm{s}}\right)_{0}=0.75$ (Wainscoat et al. 1992), and knowing that in the 2MASS observations $J_{\text {comp }}$ varies between $m_{J} \sim 12.0$ and 15.8 we can calculate that the $A_{K s(\max )}$ varies between $\sim 1.4$ and $\sim 3.75$. From equation Eq. (11), it is obvious that the highest extinction detectable will be limited by the completeness in the $J$ band alone.

For a colour difference between 2MASS and the Galactic model to be significant, the difference must be greater than the uncertainty in the $\left(J-K_{\mathrm{s}}\right)$ colour index in the observations. This sets a limit on the minimum extinction detectable. The photometric errors in the 2MASS observations depend on the Galactic region observed as well as on the local atmospheric conditions on the night the observations were made. An uncertainty of $0.05 \mathrm{mag}$ for the $\left(J-K_{\mathrm{S}}\right)$ colour index is a suitable guide, which corresponds to a minimum $K_{\mathrm{s}}$ band extinction of $\sim 0.03 \mathrm{mag}$ (Eq. (4)). This error becomes relatively small for areas of high extinction, which makes our method ideal for extinction studies in the Galactic Plane.

\subsubsection{Maximum and minimum distances}

In order to allocate a distance to a bin of stars from the 2MASS survey, we make use of the colour distance relation discussed in Sect. 4.1. To do this we need to exclude the dwarf stars from our pipeline. As these stars are local, we unfortunately lose most of the information on the first kiloparsec.

The maximum distance varies according to the $J$ and $K_{\mathrm{S}}$ completeness and column density along the line of sight. Generally, we are able to detect the extinction to distances greater than $10 \mathrm{kpc}$, and for many lines of sight well beyond this, but for lines of sight with high column densities and low completeness (central regions, spiral arm tangents) we may only obtain information out to $\sim 7 \mathrm{kpc}$. We also notice that lines of sight which contain small number of stars (large absolute longitudes) 
contain extinction information to rather short distances. Deeper counts with more stars would be needed to complete those lines of sight.

\section{Conclusion}

We present the first results of our extinction model in the Galactic plane. The Galactic model provides an excellent tool for extracting the reddening information locked in the 2MASS data.

Our method does not give much information on the extinction in the first kiloparsec from the Sun but does give the large scale distribution of the extinction in our Galaxy. Furthermore, the $J-K_{\mathrm{s}}$ index is not sensitive to very low extinction zones, such as high latitude lines of sight. However, the method can easily be modified to use other colour indices, which would allow us to adapt the sensitivity to a given region or set of observations. An additional restriction is the number of stars in the observations for our chosen pixel size. The map could thus be expanded to higher Galactic latitudes by lowering the spatial resolution.

Many structures have been identified in the resulting maps, including: several spiral arm tangents, dust in the Galactic bar, the molecular ring as well as local features such as the Aquila Rift. We have given quantitative results for three of these structures:

1. The dust distribution is found to be asymmetrically warped, in agreement with $\mathrm{CO}$ and HI observations of the ISM but not as pronounced: for positive longitudes the angle is $89^{\circ}$, it starts at $8.7 \mathrm{kpc}$ from the Galactic center and grows with a slope of 0.14 , while at negative longitudes, the angle of the maximum is at $272^{\circ}$, the starting radius $7.8 \mathrm{kpc}$ and the slope 0.11 . Hence we confirm that the warp is seen in all Galactic components, although the amplitudes appear to vary from one component to the other.

2. By converting our extinction map between 4 and $8.5 \mathrm{kpc}$ to a map of hydrogen density, we are able to calculate the mean scale height for the interstellar matter in the inner Galaxy. We find the scale height of the disc to be $125_{-7}^{+17} \mathrm{pc}$ in the region $4<R<8.5 \mathrm{kpc}$. This value of the scale height is in agreement with Drimmel et al. (2003) who find a value of $134.4 \pm 8.5 \mathrm{pc}$. However the dust scale height appears to be slightly smaller than the HI layer, as seen by Malhotra (1995) and Nakanishi \& Sofue (2003) who find a mean value of $\sim 160 \mathrm{pc}$ for the same region.

3. The bulge region is found to contain little absorbing matter apart from an elongated structure $5.2 \mathrm{kpc}$ long with an angle of $30 \pm 5^{\circ}$ relative to the Sun-Galactic centre direction. This dusty bar has a greater inclination than many measures of the angle for the stellar bar which may indicate the presence of dust lanes preceding the stellar bar at positive longitudes and trailing it at negative longitudes.

In the near future, this 3D map of the location of obscuring dust in the Milky Way will enable us to constrain dust parameters (temperature, size) by comparing the predicted far infrared emission with observations. In addition, this map of the Galactic ISM enables us to further constrain the Galactic structure parameters via the Galactic model. In particular, the spiral structure, the Galactic flare and warp, as well as the size and orientation of the bulge and stellar bar will be easier to model with this improved estimation of the three dimensional distribution of interstellar extinction.

Acknowledgements. We would like to thank the anonymous referee for the very helpful comments we received, which allowed us to improve the final version of this paper. Thanks also to Anthony Jones and François Boulanger at the Institut d'Astrophysique Spatiale for their comments and suggestions.

This publication makes use of data products from the Two Micron All Sky
Survey, which is a joint project of the University of Massachusetts and the Infrared Processing and Analysis Center/California Institute of Technology, funded by the National Aeronautics and Space Administration and the National Science Foundation. The CDSClient package, available from http://cdsweb.u-strasbg.fr/doc/ cdsclient.html, was used for the remote querying of the 2 MASS dataset. M. Schultheis was supported by an APART fellowship.

\section{References}

Arce, H. G., \& Goodman, A. A. 1999, ApJ, 512, 135

Arenou, F., Grenon, M., \& Gómez, A. 1992, A\&A, 258, 104

Babusiaux, C., \& Gilmore, G. 2005, MNRAS, 358, 1309

Berdnikov, L. N., Ibragimov, M. A., \& Pavlovskaya, E. D. 1990, Sov. Astron. Lett., 16, 308

Bertelli, G., Bressan, A., Chiosi, C., Fagotto, F., \& Nasi, E. 1994, A\&AS, 106, 275

Bissantz, N., \& Gerhard, O. 2002, MNRAS, 330, 591

Bohlin, R. C., Savage, B. D., \& Drake, J. F. 1978, ApJ, 224, 132

Bruzual, G., Barbuy, B., Ortolani, S., et al. 1997, AJ, 114, 1531

Burton, W. B. 1988, Galactic and Extragalactic Radio Astronomy, 2nd version (Springer-Verlag), 295

Cambrésy, L. 1999a, A\&A, 345, 965

Cambrésy, L. 1999b, Proceedings of Star Formation 1999, held in Nagoya, Japan, June 21-25, 1999, ed. T. Nakamoto, Nobeyama Radio Observatory, 90

Cambrésy, L., Jarrett, T. H., \& Beichman, C. A. 2005, A\&A, 435, 131

Calbet, X., Mahoney, T., Hammersley, P. L., Garzon, F., \& Lopez-Corredoira, M. 1996, ApJ, 457, L27

Chiappini, C., Matteucci, F., \& Romano, D. 2001, ApJ, 554, 1044

Cutri, R. M., Skrutskie, M. F., van Dyk, S., et al. 2003, Explanatory Supplement to the 2MASS All Sky Data Release

Dame, T. M., Hartmann, D., \& Thaddeus, P. 2001, ApJ, 547, 792

Derrière, S., \& Robin, A. C. 2001, The New era of Wide Field Astronomy, ASP Conf. Ser., 232, 229

Derrière, S. 2001, Thèse de doctorat, Université Louis Pasteur, Strasbourg

Drimmel, R., \& Spergel, D. N. 2001, ApJ, 556, 181

Drimmel, R., Cabrera-Lavers, A., \& López-Corredoira, M. 2003, A\&A, 409, 205

Dutra, C. M., Santiago, B. X., Bica, E. L. D., \& Barbuy, B. 2003, MNRAS, 338, 253

Dutra, C. M., Santiago, B. X., \& Bica, E. 2002, A\&A, 381, 219

Einasto, J. 1979, The Large Scale Characteristics of the Galaxy, ed. W. B. Burton, IAU Symp., 84, 451

Faison, M. D., \& Goss, W. M. 2001, AJ, 121, 2706

Fitzgerald, M., \& Pini 1968, AJ, 73, 983

Froebrich, D., Ray, T. P., Murphy, G. C., \& Scholz, A. 2005, A\&A, 432, L67

Gerhard, O. E., 2001, Galaxy Disks and Disk Galaxies, ASP Conf. Ser., 230, 21

Girardi, L., Bertelli, G., Bressan, A., et al. 2002, A\&A, 391, 195

Glass, I. S. 1999, Handbook of infrared astronomy, Highlights in Astronomy (Cambridge University Press)

Glass, I. S., \& Schultheis, M. 2003, MNRAS, 345, 39

Glass, I. S., Catchpole, R. M., \& Whitelock, P. A. 1987, MNRAS, 227, 373

Habing, H. J. 1996, A\&A Rev., 7, 97

Hakkila, J., Myers, J. M., Stidham, B. J., \& Hartmann, D. H. 1997, AJ, 114, 2043

Indebetouw, R., Mathis, J. S., Babler, B. L., et al. 2005, ApJ, 619, 931

Jahreiß, H., \& Wielen, R. 1997, ESA SP-402: Hipparcos - Venice '97, 675

Jones, T. J., Hyland, A. R., \& Bailey, J. 1984, ApJ, 282, 675

Jura, M., \& Kleinmann, S. G. 1989, ApJ, 341, 359

Lada, C. J., Lada, E. A., Clemens, D. P., \& Bally, J. 1994, ApJ, 429, 694

Le Bertre, T., Tanaka, M., Yamamura, I., \& Murakami, H. 2003, A\&A, 403, 943

Levine, E., Blitz, L., \& Heiles, C. 2006, ApJ, accepted

López-Corredoira, M., Cabrera-Lavers, A., Garzón, F., \& Hammersley, P. L. 2002, A\&A, 394, 883

Malhotra, S. 1995, ApJ, 448, 138

Mathis, J. S. 1990, ARA\&A, 28, 37

Nakanishi, H., \& Sofue, Y. 2003, PASJ, 55, 191

Nishiyama, Nagata, T., Kusakabe, N., et al. 2006, ApJ, 638, 839

Neckel, Th., \& Klare, G. 1980, A\&AS, 42, 251

Press, W., Teukolsky, S., Vetterling, W., \& Flannery, B. 1992, Numerical recipes in Fortran 77: the art of scientific computing, 2nd ed., 616

Picaud, S., \& Robin, A. C. 2004, A\&A, 428, 891

Rieke, G. H., \& Lebofsky, M. J. 1985, ApJ, 288, 618

Robin, A. C., Reylé, C., Derrière, S., \& Picaud, S. 2003, A\&A, 409, 523. Erratum 2004, A\&A, 416, 157

Schlegel, D. J., Finkbeiner, D. P., \& Davis, M. 1998, ApJ, 500, 525

Schultheis, M., Ganesh, S., Simon, G., et al. 1999, A\&A, 349, L69

Sumi, T. 2004, MNRAS, 349, 193

Wainscoat, R. J., Cohen, M., Volk, K., Walker, H. J., \& Schwartz, D. E. 1992, ApJS, 83,111 